\title{
Vortex motion in shallow water with varying bottom topography and zero Froude number
}

\author{
By G. RICHARDSON $\dagger$ \\ Laboratoire de Physique Statistique, Ecole Normale Supérieure, 75231 Paris Cedex 05, France
}

(Received 15 December 1998 and in revised form 29 July 1999)

The methods of formal matched asymptotics are used to investigate the motion of a vortex in shallow inviscid fluid of varying depth and zero Froude number in the limit as the vortex core radius tends to zero. To leading order the vortex is driven by local gradients in the logarithm of the depth along an isobath (or depth contour). A further term in the vortex velocity is calculated in which effects arising from the global bottom topography, other vortices and the vortex core structure appear. The evolution of the vortex core structure is then calculated. A point-vortex model is formulated which describes the motion of a number of small vortices in terms of the bottom topography, the inviscid flows around the vortices and their evolving core structure. A numerical method for solving this model is discussed and finally some numerical simulations of the motion of vortex pairs over a varying bottom topography are presented.

\section{Introduction}

In this paper we investigate the effects of a varying topography on the motion of small vortices in a surrounding shallow, inviscid, irrotational fluid. By small we mean that the typical separation between vortices and the typical lengthscale for variations in the topography are much greater than the width of the core of the vortex, within which the vorticity is contained (see figure 1). We make use of a model discussed in $\S 7.7$ of Batchelor (1967) and more recently, among others, in Camassa, Holm \& Levermore (1996) where it is termed the lake model. The assumptions made in deriving this model are: (i) vertical fluid velocities are small in comparison with horizontal velocities; (ii) the flow aspect ratio $v$, which is obtained by dividing the typical depth by the typical lengthscale for horizontal variations (in depth and fluid velocity), is small; (iii) the Froude number $\mathscr{F}$ of the flow is small (thus the free surface of the fluid is, to leading order, flat). Under this set of assumptions the Euler equations may be approximated by the following system:

$$
\begin{gathered}
\boldsymbol{u}_{t}+(\boldsymbol{u} \cdot \nabla) \boldsymbol{u}=-\nabla p, \\
\nabla \cdot(b \boldsymbol{u})=0 .
\end{gathered}
$$

Here $\boldsymbol{u}=u_{1}(x, y, t) \boldsymbol{e}_{x}+u_{2}(x, y, t) \boldsymbol{e}_{y}$ is the horizontal fluid velocity, $b(x, y)$ is the dimensionless depth of fluid and $p$ is an effective pressure which may be equated with small height variations in the free surface. We find it useful to write equation (1.1) in

$\dagger$ Present address: Department of Mathematics, University of Nottingham, Nottingham NG7 2RD, UK. 


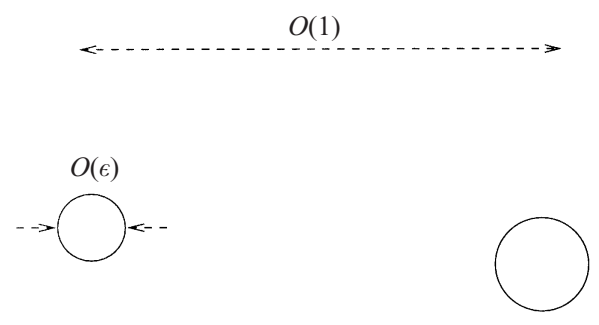

FiguRE 1. A typical configuration of vortices. The regions of significant vorticity occur within the circles (vortices) which have a typical width $O(\epsilon)$ (where $\epsilon \ll 1$ ). The typical separation between vortices and the typical lengthscale for variations on the depth is of $O(1)$.

terms of the vorticity $\omega=\nabla \wedge \boldsymbol{u}=\omega \boldsymbol{e}_{3}$ by taking its curl. This gives the following law:

$$
\omega_{t}+\nabla \cdot(\omega \boldsymbol{u})=0 .
$$

Experimental evidence for the generation of vortex-like structures in shallow water has been given by Oltman-Shay, Howd \& Birkemeier (1989) who studied the effect of a current flowing along a beach. Evidence for the generation of vortices (in shallow water) by flow round an obstacle can be found in Van Dyke (1982) which contains an aerial photograph of the flow around a grounded tanker. The marker used to visualize the flow is the oil seeping from the tanker. In Hamm, Masden \& Peregrine (1993) observations of an intermittent rip current consisting of vortex pairs propagating seaward is reported. They also remark upon the difficulty of observing large-scale flows without the presence of a marker, such as oil or sediment. Such difficulties led Couder \& Basdevant (1986) to experimentally investigate two-dimensional high Reynolds number flows in a soap film forced by a moving disc. The results of this experiment show the generation of coherent vortices and vortex couples.

The motion of small vortices in a surrounding irrotational inviscid fluids has been studied previously by Moore \& Saffman (1972) who derived the leading term in the velocity of a thin curved vortex filament using a force balance method. Subsequently Callegari \& Ting (1978) generalized this analysis to include viscous effects in the core. At the the same time they introduced a method based upon matched asymptotic expansions to replace the force balance of Moore \& Saffman. This has the advantage that it allows tracking of the evolving core structure and hence the calculation of the next term in the vortex velocity.

Numerical simulations of longshore currents, which use a shallow water equation with turbulent diffusion and bottom friction, have been conducted by Allen, Newberger \& Holman (1996) and Slinn et al. (1998). In certain parameter regimes both simulations show the shedding of vortices from the inshore region. Numerical simulations of vortex formation on headlands swept by a tidal flow have been carried out in Signell \& Geyer (1991). These also show the shedding of vortices from the headland.

In the next section we apply Callegari \& Ting's method to the model given in equations (1.1)-(1.3) and calculate the law of motion of a vortex with small dimensionless radius $\epsilon$ in shallow water with varying depth $b$. Here $\epsilon$ is the ratio 
of the typical dimensional vortex radius to the lengthscale associated with variations in the depth. We also assume that the typical separation between vortices is of the same order as the lengthscale for variations in the depth. The use of the model (1.1)-(1.3) implies that we take the limits of the Froude number $\mathscr{F} \rightarrow 0$ and the flow aspect ratio $v \rightarrow 0$ before taking the limit $\epsilon \rightarrow 0$ (i.e. $\mathscr{F} \ll \epsilon \ll 1$ and $v \ll \epsilon$ ). At leading order we find that the motion is driven by the gradient in $\log b$ (the vortex moves with speed $O(\log (1 / \epsilon))$ along a contour of $b)$. At next order $O(1)$ the vortex 'feels' other vortices and the global topography. In order to quantify this effect we need to solve an equation for the fluid flow (on the $O(1)$ lengthscale) in which vortices appear as point singularities. Our analysis bears some similarity to that carried out in Chapman \& Richardson (1997) for so-called superconducting vortices in inhomogeneous superconductors. There the leading-order term in the vortex velocity is such as to drive vortices towards regions where superconductivity is least energetically favourable (the analogue of a local minimum in the depth $b$ ). However the size of this term is again of $O(\log (1 / \epsilon))$ where $\epsilon$ gives a measure of the width of the vortex.

In $\S 2.1$ we proceed to calculate the evolution of the vortex core structure. With knowledge of the initial core structure we can then extend the instantaneous law of motion derived previously to all times; this is our main result and is given in equation (2.90). In $\S 2.3$ we summarize the results and show how, given an initial distribution of vortices (separated by $O(1)$ distances), their initial core structures, and the topography $b(\boldsymbol{x})$, we can track the subsequent motion using the simplified 'point vortex' model we derive from equations (1.1) and (1.2). In $\S 3$ and $\S 4$ we do so, presenting some analytic and numerical simulations of the motion of vortex pairs over a varying bottom topography. Finally we present our conclusions.

\section{Calculation of the vortex motion}

We consider a small patch of vorticity of width $O(\epsilon)$ (here $\epsilon \ll 1)$ centred on the point $\boldsymbol{x}=\boldsymbol{q}(t)$. In addition we assume that this vortex is well-separated (by $O(1)$ distances) from other regions of vorticity and that the induced circulation is of size $O(1)$.

The elementary solution for an isolated vortex in fluid of constant depth $b$ has the form

$$
\omega=\omega(r) \quad \boldsymbol{u}=\frac{\boldsymbol{e}_{\theta}}{r} \int_{0}^{r}\left(r^{\prime} \omega\right) \mathrm{d} r^{\prime},
$$

where $(r, \theta)$ are polar coordinates. Given that the depth of fluid is approximately constant across the width of the vortex patch and that it is well-separated from other regions of vorticity, we expect to be able to approximate its core structure by a solution of the form (2.1). However before further considering the core region (which we shall term the inner region) we look at the region surrounding the vortex, the far-field region.

Far field. Away from the vortex we assume the vorticity is small, $\omega=o(1)$. Denoting the solution in this region by the subscript $o$ we find the following equations for the velocity $\boldsymbol{u}_{o}=\boldsymbol{u}_{o}^{(0)}+\cdots$ at leading order:

$$
\begin{gathered}
\nabla \wedge \boldsymbol{u}_{o}^{(0)}=0, \\
\nabla \cdot\left(b \boldsymbol{u}_{o}^{(0)}\right)=0 .
\end{gathered}
$$


It is helpful to formulate this problem both in terms of a stream function $\psi^{(0)}$

$$
\begin{gathered}
\boldsymbol{u}_{o}^{(0)}=\frac{1}{b}\left(\psi_{y}^{(0)} \boldsymbol{e}_{x}-\psi_{x}^{(0)} \boldsymbol{e}_{y}\right), \\
\nabla \cdot\left(\frac{1}{b} \nabla \psi^{(0)}\right)=0,
\end{gathered}
$$

and in terms of a scalar potential $\phi^{(0)}$

$$
\begin{gathered}
\boldsymbol{u}_{o}^{(0)}=\nabla \phi^{(0)}, \\
\nabla \cdot\left(b \nabla \phi^{(0)}\right)=0 .
\end{gathered}
$$

Local coordinates. At any instant $t$ we may introduce local polar coordinates $(\rho, \theta)$ centred on the vortex (and the corresponding Cartesian coordinates $(\xi, \eta)$ ) such that

$$
\begin{gathered}
\boldsymbol{x}=\boldsymbol{q}(t)+\rho \cos \theta \boldsymbol{e}_{1}+\rho \sin \theta \boldsymbol{e}_{2}, \\
\xi=\rho \cos \theta, \quad \eta=\rho \sin \theta, \\
t=T .
\end{gathered}
$$

The orientation of this coordinate system is arbitrary so that, at this particular instant, we may choose to orient $\boldsymbol{e}_{1}$ parallel to the gradient in the depth $\left.\nabla b\right|_{x=q(t)}$ and $\boldsymbol{e}_{2}$ perpendicular to this. The purpose of the calculation which follows is to use these local coordinates to calculate the instantaneous velocity $\boldsymbol{v}$ of the vortex to the leading two orders, and the instantaneous evolution of the leading-order vorticity $\omega_{i}^{(0)}$ at time $t$ in terms of these coordinates. These quantities can then be related to a fixed coordinate system $(x, y)$ and, since our analysis holds for every instant $t$, the result can be used to track the vortex over $O(1)$ times.

We also find it helpful to introduce a local fluid velocity $\tilde{\boldsymbol{u}}$, in the reference frame of the vortex, which is defined as follows:

$$
\tilde{\boldsymbol{u}}=\boldsymbol{u}-\dot{\boldsymbol{q}}(t) .
$$

Inner region. We define inner coordinates by introducing the stretched variable $R$, such that $\rho=\epsilon R$. In terms of these inner coordinates equations (1.2) and (1.3) become

$$
\begin{gathered}
\epsilon \frac{\partial \omega_{i}}{\partial T}+\frac{1}{R} \frac{\partial}{\partial R}\left(R \omega_{i} \tilde{u}_{i R}\right)+\frac{1}{R} \frac{\partial}{\partial \theta}\left(\omega_{i} \tilde{u}_{i \theta}\right)=0, \\
\left(\dot{\boldsymbol{q}} \cdot \boldsymbol{e}_{R}\right) \frac{\partial b}{\partial R}+\frac{\left(\dot{\boldsymbol{q}} \cdot \boldsymbol{e}_{\theta}\right)}{R} \frac{\partial b}{\partial \theta}+\frac{1}{R} \frac{\partial}{\partial R}\left(R b \tilde{u}_{i R}\right)+\frac{1}{R} \frac{\partial}{\partial \theta}\left(b \tilde{u}_{i \theta}\right)=0, \\
\omega_{i}=\frac{1}{\epsilon}\left(\frac{1}{R} \frac{\partial}{\partial R}\left(R \tilde{u}_{i \theta}\right)-\frac{1}{R} \frac{\partial \tilde{u}_{i R}}{\partial \theta}\right) .
\end{gathered}
$$

Motivated by the elementary solution (2.1) for an isolated vortex in constant depth, we make the solution ansatz

$$
\left.\begin{array}{rl}
\omega_{i} & \sim \frac{1}{\epsilon^{2}} \omega_{i}^{(0)}(R, \tau)+\cdots, \\
\tilde{\boldsymbol{u}}_{i} & \sim \frac{\tilde{u}_{i \theta}^{(0)}(R, \tau)}{\epsilon} \boldsymbol{e}_{\theta}+\cdots,
\end{array}\right\}
$$

together with the assumption that the vorticity $\omega_{i}=o\left(1 / R^{2}\right)$ as $R \rightarrow \infty$. Here

$$
\tau=\chi(\epsilon) T,
$$


where $\chi(\epsilon)$ is the (as yet undetermined) timescale for variations in the core structure. We now substitute the above expansion into (2.12)-(2.14) to find

$$
\tilde{u}_{i \theta}^{(0)}=\frac{1}{R} \int_{0}^{R}\left(R^{\prime} \omega_{i}^{(0)}\left(R^{\prime}, \tau\right)\right) \mathrm{d} R^{\prime} .
$$

Far field. Matching the leading-order inner solution to the outer region away from the vortex core we find that

$$
\boldsymbol{u}_{o}^{(0)} \sim \frac{\Gamma \boldsymbol{e}_{\theta}}{\rho}+\cdots \quad \text { as } \quad \rho \rightarrow 0,
$$

where

$$
\Gamma=\int_{0}^{\infty}\left(R \omega_{i}^{(0)}\right) \mathrm{d} R
$$

It follows that $\phi^{(0)}$, the scalar potential for $\boldsymbol{u}_{o}^{(0)}$, also has a singularity as the vortex is approached:

$$
\phi^{(0)} \sim \Gamma \theta+\cdots \quad \text { as } \rho \rightarrow 0 .
$$

In order to determine further terms in the expansion of $\boldsymbol{u}_{o}^{(0)}$ as $\rho \rightarrow 0$ we write equation (2.7) for the outer scalar potential in terms of inner variables and expand in powers of $\epsilon$. We find that $\phi^{(0)}$ should be expanded as follows:

$$
\phi^{(0)}=\Gamma \theta+(\epsilon \log \epsilon) \Phi_{1}+\epsilon \Phi_{2}+\cdots .
$$

The $O(\epsilon \log \epsilon)$ term arises because the leading-order singularity in $\phi^{(0)}$ combined with the non-uniform depth induces a secondary $\rho \log \rho$ singularity as the vortex is approached. Substituting this expansion into (2.7) gives

$$
\begin{gathered}
\frac{1}{R} \frac{\partial}{\partial R}\left(R \frac{\partial \Phi_{1}}{\partial R}\right)+\frac{1}{R^{2}} \frac{\partial^{2} \Phi_{1}}{\partial \theta^{2}}=0 \\
\frac{1}{R} \frac{\partial}{\partial R}\left(R \frac{\partial \Phi_{2}}{\partial R}\right)+\frac{1}{R^{2}} \frac{\partial^{2} \Phi_{2}}{\partial \theta^{2}}-\frac{\Gamma}{b_{0} R} B_{1} \sin \theta=0,
\end{gathered}
$$

where we expand $b(\boldsymbol{x})$ in terms of inner coordinates

$$
\begin{aligned}
& b=b_{0}+\epsilon B_{1} R \cos \theta+\cdots, \\
& b_{0}=\left.b\right|_{x=q(t)}, \quad B_{1}=\left.b_{\xi}\right|_{x=q(t)},
\end{aligned}
$$

having chosen the coordinates $(\xi, \eta)$ such that $\left.b_{\eta}\right|_{x=q(t)}=0$. The calculation then proceeds as in Chapman \& Richardson (1997). We write

$$
\Phi_{2}=\frac{\Gamma}{2 b_{0}} B_{1} R \log R \sin \theta+\hat{\Phi}_{2},
$$

where the regular term $\hat{\Phi}_{2}$ satisfies

$$
\frac{1}{R} \frac{\partial}{\partial R}\left(R \frac{\partial \hat{\Phi}_{2}}{\partial R}\right)+\frac{1}{R^{2}} \frac{\partial^{2} \hat{\Phi}_{2}}{\partial \theta^{2}}=0 .
$$

The condition that $\phi^{(0)}$, when written in terms of outer variables, does not depend on $\epsilon$ allows us to determine $\Phi_{1}$. The expansion for $\boldsymbol{u}_{o}^{(0)}$ then follows; it is

$$
\boldsymbol{u}_{o}^{(0)} \sim \frac{\Gamma \boldsymbol{e}_{\theta}}{\rho}+\frac{\Gamma B_{1}}{2 b_{0}}\left(\log \rho \boldsymbol{e}_{2}+\sin \theta \boldsymbol{e}_{\rho}\right)+\boldsymbol{u}_{o, \text { reg }}^{(0)}+O(\rho \log \rho) \quad \text { as } \rho \rightarrow 0,
$$


where $\boldsymbol{u}_{o, r e g}^{(0)}$ is the regular part of the fluid velocity at the vortex core (i.e the part represented by the regular part of the scalar potential $\hat{\Phi}_{2}$ ). This term is determined by matching to the outer region and may be thought of as arising from the interaction of the fluid flow induced by the vortex with the global topography (as opposed to the effect of the local topography which is represented by the second term in equation (2.28)) and the effect of other vortices lying in the far field of the particular vortex in question. We can determine $\boldsymbol{u}_{o, r e g}^{(0)}$ by solving for the stream function using equation (2.5) together with its asymptotic behaviour as the vortex is approached

$$
\psi^{(0)} \sim-\Gamma b_{0} \log \rho \quad \text { as } \rho \rightarrow 0,
$$

and then comparing the result of this calculation, using the definition of the stream function contained in equation (2.4), to the asymptotic behaviour of $\boldsymbol{u}_{o}^{(0)}$ given in equation (2.28). The asymptotic behaviour of $\psi^{(0)}$ may be combined with (2.5) in the single equation

$$
\nabla \cdot\left(\frac{1}{b} \nabla \psi^{(0)}\right)=-2 \pi \Gamma \delta(\boldsymbol{x}-\boldsymbol{q}(t)) .
$$

Where several vortices (at positions $\boldsymbol{x}=\boldsymbol{q}^{i}(t)$ for $i=1, \ldots, n$ and with circulations $\Gamma_{i}$ ) interact this equation is replaced by

$$
\nabla \cdot\left(\frac{1}{b} \nabla \psi^{(0)}\right)=-2 \pi \sum_{i=1}^{n} \Gamma_{i} \delta\left(\boldsymbol{x}-\boldsymbol{q}^{i}(t)\right) .
$$

In $\S \S 3$ and 4 the calculation of solutions to equations (2.30) and (2.31) is discussed in more detail.

Inner expansion. Matching the asymptotic behaviour of $\boldsymbol{u}_{o}^{(0)}$ as $\rho \rightarrow 0$ back to the inner region, using Van Dyke's matching principle (Van Dyke 1975), we see that the inner expansion should proceed as follows:

$$
\left.\begin{array}{rl}
\omega_{i} & =\frac{\omega_{i}^{(0)}(R, \tau)}{\epsilon^{2}}+\frac{\log \epsilon}{\epsilon} \omega_{i}^{(1)}+\frac{\omega_{i}^{(2)}}{\epsilon}+\cdots, \\
\tilde{\boldsymbol{u}} & =\frac{\tilde{u}_{i \theta}^{(0)}(R, \tau)}{\epsilon} \boldsymbol{e}_{\theta}+\log \epsilon \tilde{\boldsymbol{u}}_{i}^{(1)}+\tilde{\boldsymbol{u}}_{i}^{(2)}+\cdots, \\
\dot{\boldsymbol{q}} & =(\log \epsilon) \dot{\boldsymbol{q}}^{(1)}+\dot{\boldsymbol{q}}^{(2)}+\cdots, \\
\chi(\epsilon) & =o(1 / \epsilon) .
\end{array}\right\}
$$

Substitution of the above into (2.12)-(2.14) yields, at first order,

$$
\begin{gathered}
\frac{1}{R} \frac{\partial}{\partial R}\left(R \omega_{i}^{(0)} \tilde{u}_{i R}^{(1)}\right)+\frac{1}{R} \frac{\partial}{\partial \theta}\left(\omega_{i}^{(0)} \tilde{u}_{i \theta}^{(1)}+\omega_{i}^{(1)} \tilde{u}_{i \theta}^{(0)}\right)=0, \\
\frac{1}{R} \frac{\partial}{\partial R}\left(R \tilde{u}_{i R}^{(1)}\right)+\frac{1}{R} \frac{\partial}{\partial \theta}\left(\tilde{u}_{i \theta}^{(1)}\right)=0, \\
\left(\frac{1}{R} \frac{\partial}{\partial R}\left(R \tilde{u}_{i \theta}^{(1)}\right)-\frac{1}{R} \frac{\partial \tilde{u}_{i R}^{(1)}}{\partial \theta}\right)=\omega_{i}^{(1)} .
\end{gathered}
$$

In the light of (2.34) we define a stream function $\psi_{1}$ for $\tilde{\boldsymbol{u}}_{i}^{(1)}$ such that

$$
\tilde{\boldsymbol{u}}_{i}^{(1)}=\frac{1}{R} \frac{\partial \psi_{1}}{\partial \theta} \boldsymbol{e}_{R}-\frac{\partial \psi_{1}}{\partial R} \boldsymbol{e}_{\theta}
$$


Rewriting equations (2.33) and (2.35) in terms of this stream function we find

$$
\frac{\partial \psi_{1}}{\partial \theta}\left(\frac{1}{R} \frac{\partial}{\partial R}\left(R \frac{\partial \tilde{u}_{i \theta}^{(0)}}{\partial R}\right)-\frac{\tilde{u}_{i \theta}^{(0)}}{R^{2}}\right)-\tilde{u}_{i \theta}^{(0)} \frac{\partial}{\partial \theta}\left(\frac{1}{R} \frac{\partial}{\partial R}\left(R \frac{\partial \psi_{1}}{\partial R}\right)+\frac{1}{R^{2}} \frac{\partial^{2} \psi_{1}}{\partial \theta^{2}}\right)=0 .
$$

In order to match to the outer solution (see equation (2.28)) we require that

$$
\tilde{\boldsymbol{u}}_{i}^{(1)} \sim \frac{\Gamma B_{1}}{2 b_{o}} \boldsymbol{e}_{2}-\dot{\boldsymbol{q}}_{1} \quad \text { as } \quad R \rightarrow \infty .
$$

With this motivation we look for a solution of the form

$$
\psi_{1}=g(R, \tau) \cos \theta+p(R, \tau) \cos \left(\theta-\alpha_{1}\right),
$$

where $\alpha_{1}$ is chosen to match onto $\dot{\boldsymbol{q}}_{1}$. Substituting this into (2.37), equating coefficients of $\sin \theta$ and integrating gives

$$
\frac{\partial \tilde{u}_{i \theta}^{(0)}}{\partial R} g-\frac{\partial g}{\partial R} \tilde{u}_{i \theta}^{(0)}=\frac{\text { const. }}{R}
$$

We assume that the leading-order velocity and vorticity are bounded at the origin so that

$$
\tilde{u}_{i \theta}^{(0)} \sim q(T) R \quad \text { as } \quad R \rightarrow 0,
$$

and require that the first-order correction to the velocity remain an order of magnitude smaller than this. It follows that $g=o(R)$ as $R \rightarrow 0$, and the constant on the righthand side of equation (2.40) is zero. We then solve for $g$ to find $g(R, \tau) \equiv 0$. We can use essentially the same argument to show that $p(R, \tau) \equiv 0$. Substituting these results back into (2.39) we conclude that $\psi_{1} \equiv 0$ and thus that the first-order correction to the velocity, measured in the vortex frame, is

$$
\tilde{\boldsymbol{u}}_{i}^{(1)} \equiv \mathbf{0} \text {. }
$$

Before matching with the outer expansion we proceed to determine one more term in the inner expansion. At next order substitution of (2.32) into (2.12)-(2.14) yields

$$
\begin{gathered}
\frac{1}{R} \frac{\partial}{\partial R}\left(R\left(\omega_{i}^{(0)} \tilde{u}_{i R}^{(2)}\right)\right)+\frac{1}{R} \frac{\partial}{\partial \theta}\left(\omega_{i}^{(0)} \tilde{u}_{i \theta}^{(2)}+\omega_{i}^{(2)} \tilde{u}_{i \theta}^{(0)}\right)=0 \\
\frac{\partial}{\partial R}\left(R \tilde{u}_{i R}^{(2)}\right)+\frac{\partial \tilde{u}_{i \theta}^{(2)}}{\partial \theta}-\frac{B_{1} R}{b_{0}} \tilde{u}_{i \theta}^{(0)} \sin \theta=0 \\
\frac{1}{R} \frac{\partial}{\partial R}\left(R \tilde{u}_{i \theta}^{(2)}\right)-\frac{1}{R} \frac{\partial \tilde{u}_{i R}^{(2)}}{\partial \theta}=\omega_{i}^{(2)}
\end{gathered}
$$

In the light of equation (2.44) we define a 'stream function' for $\tilde{\boldsymbol{u}}_{i}^{(2)}$ such that

$$
\tilde{\boldsymbol{u}}_{i}^{(2)}=-\frac{B_{1}}{b_{0}} R \tilde{u}_{i \theta}^{(0)} \cos \theta \boldsymbol{e}_{\theta}+\frac{1}{R} \frac{\partial \psi_{2}}{\partial \theta} \boldsymbol{e}_{R}-\frac{\partial \psi_{2}}{\partial R} \boldsymbol{e}_{\theta} .
$$

Substitution of the above into (2.43) and (2.45) gives rise to the following equation for $\psi_{2}$ :

$$
\begin{array}{r}
\frac{\partial \psi_{2}}{\partial \theta}\left(\frac{\partial^{2} \tilde{u}_{i \theta}^{(0)}}{\partial R}+\frac{1}{R} \frac{\partial \tilde{u}_{i \theta}^{(0)}}{\partial R}-\frac{\tilde{u}_{i \theta}^{(0)}}{R^{2}}\right)-\tilde{u}_{i \theta}^{(0)} \frac{\partial}{\partial \theta}\left(\frac{1}{R} \frac{\partial}{\partial R}\left(R \frac{\partial \psi_{2}}{\partial R}\right)+\frac{1}{R^{2}} \frac{\partial^{2} \psi_{2}}{\partial \theta^{2}}\right) \\
+\frac{B_{1}}{b_{0}} \tilde{u}_{i \theta}^{(0)} \sin \theta\left(\frac{\partial}{\partial R}\left(R \tilde{u}_{i \theta}^{(0)}\right)+\frac{1}{R} \frac{\partial}{\partial R}\left(R^{2} \tilde{u}_{i \theta}^{(0)}\right)\right)=0 .
\end{array}
$$


Motivated by the far-field matching condition

$$
\tilde{\boldsymbol{u}}_{i}^{(2)} \sim \frac{\Gamma B_{1}}{2 b_{0}}\left(\log R \boldsymbol{e}_{2}+\sin \theta \boldsymbol{e}_{R}\right)+\boldsymbol{u}_{, \text {reg }}^{(0)}-\dot{\boldsymbol{q}}^{(2)} \quad \text { as } \quad R \rightarrow \infty
$$

(see equation (2.28)) we look for a solution of the form

$$
\psi_{2}=h(R, \tau) \cos \theta+j(R, \tau) \cos \left(\theta-\alpha_{2}\right) .
$$

Here the second term in $\psi_{2}$ is chosen to match onto $\boldsymbol{u}_{\text {orreg }}^{(0)}$ (which we write in the form $\left.\boldsymbol{u}_{o, \text { reg }}^{(0)}=M\left(\sin \alpha_{2} \boldsymbol{e}_{1}-\cos \alpha_{2} \boldsymbol{e}_{2}\right)\right)$ in the limit $R \rightarrow \infty$. Substituting this into (2.47) and equating coefficients of $\sin \left(\theta-\alpha_{2}\right)$ we find an equation for $j(R, \tau)$ identical to $(2.40)$ but with $g$ replaced by $j$; we can thus show that $j \equiv 0$. Equating coefficients of $\sin \theta$ in (2.47) we find that

$$
\tilde{u}_{i \theta}^{(0)} \frac{\partial h}{\partial R}-h \frac{\partial \tilde{u}_{i \theta}^{(0)}}{\partial R}=-\frac{B_{1}}{b_{0} R} \int_{0}^{R}\left(2 R^{2} \tilde{u}_{i \theta}^{(0)} \frac{\partial \tilde{u}_{i \theta}^{(0)}}{\partial R}+3 R\left(\tilde{u}_{i \theta}^{(0)}\right)^{2}\right) \mathrm{d} R .
$$

Recall now that $\tilde{u}_{i \theta}^{(0)} \sim \Gamma / R$ as $R \rightarrow \infty$. It follows that

$$
\int_{0}^{R}\left(2 R^{2} \tilde{u}_{i \theta}^{(0)} \frac{\partial \tilde{u}_{i \theta}^{(0)}}{\partial R}+3 R\left(\tilde{u}_{i \theta}^{(0)}\right)^{2}\right) \mathrm{d} R \sim \Gamma^{2} \log R \quad \text { as } \quad R \rightarrow \infty,
$$

from which it can be seen that the asymptotic behaviour of $h$ is

$$
h \sim-\frac{B_{1} \Gamma}{2 b_{0}}\left(R \log R+R\left(\frac{\mathscr{P}(T)}{\Gamma^{2}}-\frac{1}{2}\right)+\cdots\right) \quad \text { as } \quad R \rightarrow \infty,
$$

where

$$
\mathscr{P}(T)=\lim _{\rho \rightarrow \infty}\left(\int_{0}^{\rho}\left(2 R^{2} \tilde{u}_{i \theta}^{(0)} \frac{\partial \tilde{u}_{i \theta}^{(0)}}{\partial R}+3 R\left(\tilde{u}_{i \theta}^{(0)}\right)^{2}\right) \mathrm{d} R-\Gamma^{2} \log \rho\right) .
$$

The asymptotic behaviour of $\tilde{\boldsymbol{u}}_{i}^{(2)}$ follows; it is

$$
\tilde{\boldsymbol{u}}_{i}^{(2)} \sim \frac{B_{1} \Gamma}{2 b_{0}}\left(\log R \boldsymbol{e}_{2}+\sin \theta \boldsymbol{e}_{R}+\left(\frac{\mathscr{P}(T)}{\Gamma^{2}}-\frac{3}{2}\right) \boldsymbol{e}_{2}\right) \quad \text { as } \quad R \rightarrow \infty .
$$

Matching. We complete the derivation of the vortex velocity by matching the farfield behaviour of the inner terms $\tilde{\boldsymbol{u}}_{i}^{(1)}$ (equation (2.42)) and $\tilde{\boldsymbol{u}}_{i}^{(2)}$ (equation (2.54)) to the expansion of the leading-order outer velocity $\boldsymbol{u}_{o}^{(0)}$ given in equation (2.28) (see Van Dyke 1975), via the relation (2.11). We find that in order for inner and outer solutions to match we require

$$
\begin{gathered}
\dot{\boldsymbol{q}}^{(1)}=\frac{B_{1} \Gamma}{2 b_{0}} \boldsymbol{e}_{2}, \\
\dot{\boldsymbol{q}}^{(2)}=\boldsymbol{u}_{o, r e g}^{(0)}-\frac{B_{1} \Gamma}{2 b_{0}}\left(\frac{\mathscr{P}(T)}{\Gamma^{2}}-\frac{3}{2}\right) \boldsymbol{e}_{2} .
\end{gathered}
$$

Remark. We can now write down the asymptotic behaviour of the fluid velocity in the fixed global frame up to the second term in the expansion:

$$
\begin{array}{r}
\boldsymbol{u}_{i}=\tilde{\boldsymbol{u}}_{i}+\dot{\boldsymbol{q}} \sim \frac{\Gamma \boldsymbol{e}_{\theta}}{\epsilon R}+\frac{B_{1} \Gamma}{2 b_{0}}\left(\log (\epsilon R) \boldsymbol{e}_{2}+\frac{1}{2}\left(\sin 2 \theta \boldsymbol{e}_{1}-\cos 2 \theta \boldsymbol{e}_{2}\right)+\frac{1}{2} \boldsymbol{e}_{2}\right) \\
+\boldsymbol{u}_{o, \text { reg }}^{(0)} \quad \text { as } \quad R \rightarrow \infty .
\end{array}
$$


Here the third term on the right-hand side of this equation represents the effect of a vortex dipole. In this sense the driving mechanism for the vortex motion seems to bear some similarity to the so-called ' $\beta$-gyre' which was identified by Sutyrin $\&$ Fleirl (1994) and Reznik \& Dewar (1994) as the driving mechanism for isolated vortices on the $\beta$-plane. The term isolated here refers to the fact that the circulation around the vortex decays to zero far from the vortex. This is an important difference between the case under consideration in this work and the vortex on the $\beta$-plane. In particular the fluid velocity for the isolated vortex on the $\beta$-plane has no logarithmic term. Furthermore if we consider an isolated vortex in this setting then $\Gamma=0$ and it would have zero self-induced velocity to the first two orders (see equations (2.55) and (2.56)).

\subsection{Evolution of the vortex core structure}

The $O(1)$ velocity of the vortex $\dot{\boldsymbol{q}}^{(2)}$ depends upon the function of time $\mathscr{P}(T)$ (see equation (2.56)) and this function itself depends upon the distribution of vorticity in the vortex core through equation (2.53). Thus, in order to accurately track the position of the vortex it is neccessary to calculate the vorticity distribution in the core at each time step. In this section we calculate the evolution of $\omega_{i}^{(0)}(R, \tau)$. Returning to the far field we look for further terms in the expansion of the leading-order velocity $\boldsymbol{u}_{o}^{(0)}$ as the vortex core is approached $(\rho \rightarrow 0)$. In order to do this we must first expand $b$ in terms of inner coordinates up to $O\left(\epsilon^{2}\right)$ :

$$
b=b_{0}+\epsilon B_{1} R \cos \theta+\epsilon^{2} R^{2}\left(\beta_{1} \cos ^{2} \theta+\beta_{2} \cos \theta \sin \theta+\beta_{3} \sin ^{2} \theta\right)+\cdots,
$$

where

$$
\beta_{1}=\left.\frac{\partial^{2} b}{\partial \xi^{2}}\right|_{x=q(t)}, \quad \beta_{2}=\left.\frac{\partial^{2} b}{\partial \xi \partial \eta}\right|_{x=q(t)}, \quad \beta_{3}=\left.\frac{\partial^{2} b}{\partial \eta^{2}}\right|_{x=q(t)} .
$$

The calculation then proceeds as in the previous section, that is we continue the expansion of $\phi^{(0)}$ in terms of inner coordinates started in equation (2.21). We find, after considerable algebra, the following expansion for the leading-order velocity as the vortex core is approached:

$$
\begin{aligned}
\boldsymbol{u}_{o}^{(0)} \sim & \frac{\Gamma \boldsymbol{e}_{\theta}}{\rho}+\frac{\Gamma B_{1}}{2 b_{0}}\left(\log \rho \boldsymbol{e}_{2}+\sin \theta \boldsymbol{e}_{\rho}\right)+\boldsymbol{u}_{o, r e g}^{(0)} \\
& -\frac{\Gamma}{2 b_{0}} \rho \log \rho\left[\beta_{2}\left(\cos 2 \theta \boldsymbol{e}_{R}-\sin 2 \theta \boldsymbol{e}_{\theta}\right)+\left(\frac{3 b_{1}^{2}}{4 b_{0}}+\beta_{3}-\beta_{1}\right)\left(\sin 2 \theta \boldsymbol{e}_{R}+\cos 2 \theta \boldsymbol{e}_{\theta}\right)\right] \\
& -\frac{\Gamma}{4 b_{0}}\left(\beta_{2} \cos 2 \theta+\left(\frac{3 b_{1}^{2}}{4 b_{0}}+\beta_{3}-\beta_{1}\right) \sin 2 \theta\right) \boldsymbol{e}_{R}-\rho \frac{\mathscr{U}_{1} B_{1}}{2 b_{0}} \boldsymbol{e}_{R} \\
& +k_{1} \rho\left(\cos 2 \theta \boldsymbol{e}_{R}-\sin 2 \theta \boldsymbol{e}_{\theta}\right)+k_{2} \rho\left(\sin 2 \theta \boldsymbol{e}_{R}+\cos 2 \theta \boldsymbol{e}_{\theta}\right)+O\left(\rho^{2} \log \rho\right) \quad \text { as } \quad \rho \rightarrow 0 .
\end{aligned}
$$

Here we write $\boldsymbol{u}_{o, \text { reg }}^{(0)}=\mathscr{U}_{1} \boldsymbol{e}_{1}+\mathscr{U}_{2} \boldsymbol{e}_{2}$; the unknown constants $k_{1}$ and $k_{2}$ are determined by solution of equation (2.31).

Inner expansion. Matching the asymptotic behaviour of $\boldsymbol{u}_{o}^{(0)}$ obtained in (2.60) to the inner region it becomes clear that there are terms in $\tilde{\boldsymbol{u}}_{i}$ at $O(\epsilon \log \epsilon)$ and $O(\epsilon)$. The 
inner expansion thus proceeds as follows:

$$
\left.\begin{array}{rl}
\omega_{i} & =\frac{\omega_{i}^{(0)}(R, \tau)}{\epsilon^{2}}+\frac{\log \epsilon}{\epsilon} \omega_{i}^{(1)}+\frac{\omega_{i}^{(2)}}{\epsilon}+\log \epsilon \omega_{i}^{(3)}+\omega_{i}^{(4)} \ldots, \\
\tilde{\boldsymbol{u}} & =\frac{\tilde{u}_{i \theta}^{(0)}(R, \tau)}{\epsilon} \boldsymbol{e}_{\theta}+\log \epsilon \tilde{\boldsymbol{u}}_{i}^{(1)}+\tilde{\boldsymbol{u}}_{i}^{(2)}+\epsilon \log \epsilon \tilde{\boldsymbol{u}}_{i}^{(3)}+\epsilon \tilde{\boldsymbol{u}}_{i}^{(4)}+\cdots, \\
\dot{\boldsymbol{q}} & =\log \epsilon \dot{\boldsymbol{q}}^{(1)}+\dot{\boldsymbol{q}}^{(2)}+\epsilon \log \epsilon \dot{\boldsymbol{q}}^{(3)}+\epsilon \dot{\boldsymbol{q}}^{(4)}+\cdots, \\
\chi(\epsilon) & =\log \epsilon \chi^{(0)}+\chi^{(1)}+\cdots,
\end{array}\right\}
$$

where $\chi(\epsilon)$ gives the timescale for the evolution of the core as defined in equation (2.16). The first- and second-order vorticities and velocities, which we derived previously, are

$$
\begin{gathered}
\tilde{\boldsymbol{u}}_{i}^{(1)}=0, \quad \omega_{i}^{(1)}=0, \\
\tilde{\boldsymbol{u}}_{i}^{(2)}=-\left(\frac{B_{1} R \tilde{u}_{i \theta}^{(0)}}{b_{0}}+\frac{\partial h}{\partial R}(R, \tau)\right) \cos \theta \boldsymbol{e}_{\theta}-\frac{h(R, \tau)}{R} \sin \theta \boldsymbol{e}_{R}, \\
\omega_{i}^{(2)}=-\left(\frac{1}{R} \frac{\partial}{\partial R}\left(\frac{B_{1} R^{2} \tilde{u}_{i \theta}^{(0)}}{b_{0}}+R \frac{\partial h}{\partial R}\right)-\frac{h}{R^{2}}\right) \cos \theta,
\end{gathered}
$$

and the leading components in the vortex velocity, $\dot{\boldsymbol{q}}^{(1)}$ and $\dot{\boldsymbol{q}}^{(2)}$, are given in (2.55) and (2.56). Substituting this expansion, together with (2.58), into (2.12)-(2.14) and expanding to the appropriate orders we find the following equations for $\tilde{\boldsymbol{u}}_{i}^{(3)}$ and $\tilde{\boldsymbol{u}}_{i}^{(4)}$ :

$$
\begin{gathered}
\chi^{(0)} \frac{\partial \omega_{i}^{(0)}}{\partial \tau}+\frac{1}{R} \frac{\partial}{\partial R}\left(R \omega_{i}^{(0)} \tilde{u}_{i R}^{(3)}\right)+\frac{1}{R} \frac{\partial}{\partial \theta}\left(\omega_{i}^{(0)} \tilde{u}_{i \theta}^{(3)}+\omega_{i}^{(3)} \tilde{u}_{i \theta}^{(0)}\right)=0, \\
\frac{1}{R} \frac{\partial}{\partial R}\left(R \tilde{u}_{i R}^{(3)}\right)+\frac{1}{R} \frac{\partial}{\partial \theta}\left(\tilde{u}_{i \theta}^{(3)}\right)=0 \\
\frac{1}{R} \frac{\partial}{\partial R}\left(R \tilde{u}_{i \theta}^{(3)}\right)-\frac{1}{R} \frac{\partial \tilde{u}_{i R}^{(3)}}{\partial \theta}=\omega_{i}^{(3)} \\
\chi^{(1)} \frac{\partial \omega_{i}^{(0)}}{\partial \tau}+\frac{1}{R} \frac{\partial}{\partial R}\left(R\left(\omega_{i}^{(0)} \tilde{u}_{i R}^{(4)}+\omega_{i}^{(2)} \tilde{u}_{i R}^{(2)}\right)\right) \\
+\frac{1}{R} \frac{\partial}{\partial \theta}\left(\omega_{i}^{(0)} \tilde{u}_{i \theta}^{(4)}+\omega_{i}^{(4)} \tilde{u}_{i \theta}^{(0)}+\omega_{i}^{(2)} \tilde{u}_{i \theta}^{(2)}\right)=0, \\
B_{1} \mathscr{U}_{1}+\frac{1}{R} \frac{\partial}{\partial R}\left(R\left(b_{0} \tilde{u}_{i R}^{(4)}+B_{1} R \cos \theta \tilde{u}_{i R}^{(2)}\right)\right)+\frac{1}{R} \frac{\partial}{\partial \theta}\left[b_{0} \tilde{u}_{i \theta}^{(4)}+B_{1} R \cos \theta \tilde{u}_{i \theta}^{(2)}\right. \\
\left.+R^{2} \tilde{u}_{i \theta}^{(0)}\left(\beta_{1} \cos { }^{2} \theta+\beta_{2} \sin \theta \cos \theta+\beta_{3} \sin ^{2} \theta\right)\right]=0, \\
\frac{1}{R} \frac{\partial}{\partial R}\left(R \tilde{u}_{i \theta}^{(4)}\right)-\frac{1}{R} \frac{\partial \tilde{u}_{i R}^{(4)}}{\partial \theta}=\omega_{i}^{(4)} .
\end{gathered}
$$

Our goal now is to calculate $\chi^{(0)}\left(\partial \omega_{i}^{(0)} / \partial \tau\right)$ and $\chi^{(1)}\left(\partial \omega_{i}^{(0)} / \partial \tau\right)$. Since these are functions of $R$ and $\tau$ only we follow Callegari \& Ting (1978) and integrate equations (2.65)(2.70) between $\theta=0$ and $\theta=2 \pi$; we find

$$
\chi^{(0)} \frac{\partial \omega_{i}^{(0)}}{\partial \tau}+\frac{1}{R} \frac{\partial}{\partial R}\left(R \omega_{i}^{(0)}\left\langle\tilde{u}_{i R}^{(3)}\right\rangle\right)=0
$$


Vortex motion in shallow water with varying bottom topography

$$
\begin{gathered}
\frac{\partial}{\partial R}\left(R\left\langle\tilde{u}_{i R}^{(3)}\right\rangle\right)=0, \\
\chi^{(1)} \frac{\partial \omega_{i}^{(0)}}{\partial \tau}+\frac{1}{R} \frac{\partial}{\partial R}\left(R \omega_{i}^{(0)}\left\langle\tilde{u}_{i R}^{(4)}\right\rangle\right)=0, \\
\frac{\partial}{\partial R}\left(R\left\langle\tilde{u}_{i R}^{(4)}\right\rangle\right)+\frac{B_{1} \mathscr{U}_{1}}{b_{0}} R=0 .
\end{gathered}
$$

Here angled brackets around a quantity indicate that it has been averaged over $\theta$ so that, for example,

$$
\langle\zeta\rangle(R)=\frac{1}{2 \pi} \int_{0}^{2 \pi} \zeta(R, \theta) \mathrm{d} \theta .
$$

Imposing the condition that fluid velocities are bounded we solve (2.71)-(2.74) to find

$$
\left\langle\tilde{u}_{i R}^{(3)}\right\rangle=0 \quad \Longrightarrow \quad \chi^{(0)}=0,
$$

and

$$
\begin{gathered}
\left\langle\tilde{u}_{i R}^{(4)}\right\rangle=-\frac{B_{1} \mathscr{U}_{1}}{2 b_{0}} R, \\
\frac{\partial \omega_{i}^{(0)}}{\partial \tau}=\frac{B_{1} \mathscr{U}_{1}}{2 b_{0}} \frac{1}{R} \frac{\partial}{\partial R}\left(R^{2} \omega_{i}^{(0)}\right),
\end{gathered}
$$

where without loss of generality we take $\chi^{(1)}=1$. By substituting this value of $\chi^{(1)}$ (together with the value of $\chi^{(0)}$ obtained in (2.76)) into the expansion (2.61) and then using equation (2.16) we can find the following relation between $T$ and $\tau$ :

$$
t=T=\tau+o(1) .
$$

In (2.78) we have an equation for the instantaneous evolution of the vorticity in the vortex core. To track the evolution over an $O(1)$ timescale we rewrite the coefficient $B_{1} \mathscr{U}_{1} /\left(2 b_{0}\right)$ in terms of outer quantities:

$$
\begin{gathered}
\frac{\partial \omega_{i}^{(0)}}{\partial \tau}=P(\tau) \frac{1}{R} \frac{\partial}{\partial R}\left(R^{2} \omega_{i}^{(0)}\right), \\
P(\tau)=\left.\left(\frac{(\dot{\boldsymbol{q}} \cdot \nabla) \log b}{2}\right)\right|_{x=q(\tau)}+o(1) .
\end{gathered}
$$

Equation (2.80) has solution

$$
\omega_{i}^{(0)}=\exp \left(2 \int_{0}^{\tau} P\left(\tau^{\prime}\right) \mathrm{d} \tau^{\prime}\right) \mathscr{G}\left(R \exp \left(\int_{0}^{\tau} P\left(\tau^{\prime}\right) \mathrm{d} \tau^{\prime}\right)\right),
$$

where $\left.\omega_{i}^{(0)}\right|_{T=0}=\mathscr{G}(R)$. Combining this solution with the expression (2.81) for $P(\tau)$ we see that the leading-order vorticity may be expressed as follows over $O(1)$ timescales:

$$
\omega_{i}^{(0)}(R, T)=\frac{b(\boldsymbol{q}(T))}{b(\boldsymbol{q}(0))} \mathscr{G}\left(R\left(\frac{b(\boldsymbol{q}(T))}{b(\boldsymbol{q}(0))}\right)^{1 / 2}\right) .
$$

Remark. Equation (1.3) implies the conservation of relative global vorticity such that

$$
\frac{\mathrm{d}}{\mathrm{d} t} \iint_{A^{\prime}} \omega \mathrm{d} A=0
$$

where $A^{\prime}$ is some region whose boundary is convected with the fluid velocity $\boldsymbol{u}$. It is thus reassuring to note that equation (2.83) implies that the total relative vorticity (to 
leading order) contained within the vortex core is conserved. That is to say

$$
\begin{aligned}
\int_{0}^{\infty}\left(\omega_{i}^{(0)} R\right) \mathrm{d} R & =\int_{0}^{\infty}\left[\frac{b(\boldsymbol{q}(T))}{b(\boldsymbol{q}(0))} \mathscr{G}\left(\left(\frac{b(\boldsymbol{q}(T))}{b(\boldsymbol{q}(0))}\right)^{1 / 2} R\right) R\right] \mathrm{d} R \\
& =\int_{0}^{\infty}(\mathscr{G}(\varsigma) \varsigma) \mathrm{d} \varsigma=\text { const. }
\end{aligned}
$$

In fact the rearrangement of the core structure is due to $\boldsymbol{u}$ being non-divergence free. Thus as the vortex moves to shallower water, such that $\nabla \cdot \boldsymbol{u}>0$ in the inner region, the vorticity distribution undergoes a uniform dilation consistent with (2.84) while as the vortex moves to deeper water, such that $\nabla \cdot \boldsymbol{u}<0$ in the inner region, it undergoes a uniform contraction. $\dagger$

\subsection{The effect of the evolution of the core on the vortex motion}

The motion of the vortex depends on the core structure through the parameter $\mathscr{P}(T)$ given in equation (2.53). Writing the leading-order vorticity in the form

$$
\omega_{i}^{(0)}=\mu^{2}(T) \mathscr{G}(\mu(T) R) \quad \text { where } \quad \mu(T)=\left(\frac{b(\boldsymbol{q}(T))}{b(\boldsymbol{q}(0))}\right)^{1 / 2},
$$

allows us to express the leading-order velocity as follows:

$$
\tilde{u}_{i \theta}^{(0)}=\frac{1}{R} \int_{0}^{\mu(T) R} A \mathscr{G}(A) \mathrm{d} A=\frac{1}{R} \zeta(\mu(T) R) .
$$

Substituting the above into (2.53) we find that $\mathscr{P}(T)$ may be split into time-dependent and time-independent parts such that

$$
\mathscr{P}(T)=\tilde{P}+\Gamma^{2} \log (\mu(T)),
$$

where

$$
\tilde{\mathscr{P}}=\lim _{A \rightarrow \infty}\left(\int_{0}^{A}\left[\left(\frac{\zeta^{2}\left(A^{\prime}\right)}{A^{\prime}}+2 \zeta\left(A^{\prime}\right) \frac{\mathrm{d} \zeta\left(A^{\prime}\right)}{d A^{\prime}}\right)\right] \mathrm{d} A^{\prime}-\Gamma^{2} \log A\right) .
$$

Then, by referring back to equations (2.55) and (2.56), we find the vortex velocity can be written in the following form:

$$
\dot{\boldsymbol{q}}(T)=\left.\frac{\Gamma}{2}\left(\log \left(\frac{\mu(T)}{\epsilon}\right)+\frac{\tilde{P}}{\Gamma^{2}}-\frac{3}{2}\right) \nabla \log b\right|_{x=q(T)} \wedge \boldsymbol{e}_{3}+\boldsymbol{u}_{o, r e g}^{(0)}+O(\epsilon \log \epsilon) .
$$

Here $\boldsymbol{u}_{o, \text { reg }}^{(0)}$ is the regular part of the fluid velocity at the vortex. It may be calculated by solving for the stream function $\psi^{(0)}$ using equation (2.31), calculating $b \boldsymbol{u}_{o}^{(0)}$ using (2.4), expanding $\boldsymbol{u}_{o}^{(0)}$ and then comparing the result with the expansion in (2.28).

\subsection{Summary of the results and a prescription for calculating the vortex velocity}

In this section we have looked at vortices in a background inviscid flow, in shallow water of varying depth $b(x, y)$ and at zero Froude number. We assumed the typical non-dimensional width of the vortices to be of $O(\epsilon)$ which is small $(\epsilon \ll 1)$ in comparison with the typical separation between vortices and the lengthscale for variations in the depth, which we assumed are both $O(1)$ quantities. Because of the

$\dagger$ Providing the time-dependent typical lengthscale of the vortex, as measured in the outer region, $\epsilon(b(\boldsymbol{q}(T)) / b(\boldsymbol{q}(0)))^{1 / 2}$ remains $o(1)$ the asymptotics remain valid. 
difference in the lengthscales we were able to write down the leading-order equation for the inviscid fluid flow on the long $O(1)$ lengthscale, in terms of a stream function $\psi^{(0)}$ :

$$
\begin{gathered}
\boldsymbol{u}_{o}^{(0)}=\frac{1}{b}\left(\psi_{y}^{(0)} \boldsymbol{e}_{x}-\psi_{x}^{(0)} \boldsymbol{e}_{y}\right), \\
\nabla \cdot\left(\frac{1}{b} \nabla \psi^{(0)}\right)=-2 \pi \sum_{i=1}^{n} \Gamma_{j} \delta\left(\boldsymbol{x}-\boldsymbol{q}^{j}(t)\right) .
\end{gathered}
$$

Here the 'singular terms' on the right-hand side of (2.92) represent the contributions of $n$ vortices to the surrounding inviscid flow, where the $j$ th vortex is at position $\boldsymbol{x}=\boldsymbol{q}^{j}(t)$ and has a circulation $\Gamma_{j}$. At any particular instant of time it is possible to solve (2.92) to find the flow and make a local expansion of $\boldsymbol{u}_{o}^{(0)}$ about each of the vortices in turn. In order to do this we defined local coordinates $(\rho, \theta)$ about $\boldsymbol{x}=\boldsymbol{q}^{j}$ say (the position of the $j$ th vortex) such that the depth had the following expansion about this point:

$$
b=b_{0}+B_{1} \rho \cos \theta+\cdots, \quad \nabla(\rho \cos \theta)=\boldsymbol{e}_{1}, \quad \nabla(\rho \sin \theta)=\boldsymbol{e}_{2} .
$$

In other words we chose $e_{1}$ to lie parallel to the local gradient in the depth $b$ at the point $\boldsymbol{x}=\boldsymbol{q}^{j}$. We then found that the local expansion of the fluid velocity about the $j$ th vortex has the following general form:

$$
\boldsymbol{u}_{o}^{(0)} \sim \frac{\Gamma_{j} \boldsymbol{e}_{\theta}}{\rho}+\frac{\Gamma_{j} B_{1}}{2 b_{0}}\left(\log \rho \boldsymbol{e}_{2}+\sin \theta \boldsymbol{e}_{\rho}\right)+\left(\boldsymbol{u}_{o, r e g}^{(0)}\right)_{j}+O(\rho \log \rho) \quad \text { as } \quad \rho \rightarrow 0 .
$$

The unknown term $\left(\boldsymbol{u}_{o, r e g}\right)_{j}$ can be computed by comparing this expansion to the expansion of the solution to (2.92) and (2.91) in the vicinity of $\boldsymbol{x}=\boldsymbol{q}^{j}$. The velocity of the $j$ th vortex can then be computed to the leading two orders of magnitude by using the vortex law of motion we derived in equation (2.90), namely

$$
\begin{aligned}
\dot{\boldsymbol{q}}_{j}(T)= & \left.\frac{\Gamma_{j}}{2}\left(\log \left(\frac{1}{\epsilon}\left(\frac{b\left(\boldsymbol{q}_{j}(T)\right)}{b\left(\boldsymbol{q}_{j}(0)\right)}\right)^{1 / 2}\right)+\frac{\tilde{\mathscr{P}}_{j}}{\Gamma_{j}^{2}}-\frac{3}{2}\right) \nabla \log b\right|_{\boldsymbol{x}=\boldsymbol{q}^{j}(T)} \wedge \boldsymbol{e}_{3} \\
& +\left(\boldsymbol{u}_{o, \text { reg }}^{(0)}\right)_{j}+O(\epsilon \log \epsilon) .
\end{aligned}
$$

Here $\tilde{P}_{j}$ represents the effect of the vortex core structure upon the motion and where the initial profile of the vorticty in the core is given by

$$
\left.\omega_{i}^{(0)}\right|_{T=0}=\mathscr{G}_{j}(R) \quad(\rho=\epsilon R) .
$$

$\tilde{P}_{j}$ is defined as follows (see equations (2.87) and (2.89)):

$$
\begin{gathered}
\zeta_{j}(A)=\int_{0}^{A}\left(A \mathscr{G}_{j}(A)\right) \mathrm{d} A \\
\tilde{\mathscr{P}}_{j}=\lim _{A \rightarrow \infty}\left(\int_{0}^{A}\left[\frac{\zeta_{j}^{2}\left(A^{\prime}\right)}{A^{\prime}}+2 \zeta_{j}\left(A^{\prime}\right) \frac{\mathrm{d} \zeta_{j}\left(A^{\prime}\right)}{\mathrm{d} A^{\prime}}\right] \mathrm{d} A^{\prime}-\Gamma_{j}^{2} \log A\right) .
\end{gathered}
$$

\section{An analytical solution to the model}

In this section we illustrate the law of motion for a special case for which an analytic solution exists to the far-field flow problem described by equations (2.91)-(2.92) (this 
solution was first given by Grimshaw \& Yi 1991). $\dagger$ We consider the following depth profile:

$$
b=H \exp (-\alpha x) .
$$

We then seek a solution to equation (2.30) for the far-field inviscid flow around a single vortex by writing the stream function in the form

$$
\psi^{(0)}=\exp \left(-\frac{\alpha x}{2}\right) \phi(x, y) .
$$

Substituting the above into equation (2.30) we find a modified Helmholtz equation for $\phi$,

$$
\nabla^{2} \phi-\left(\frac{\alpha}{2}\right)^{2} \phi=-2 \pi \Gamma H \exp \left(-\frac{\alpha x}{2}\right) \delta\left(x-q_{1}\right) \delta\left(y-q_{2}\right),
$$

with solution

$$
\phi=\Gamma H \exp \left(-\frac{\alpha q_{1}}{2}\right) K_{0}\left(\frac{\alpha}{2}|\boldsymbol{x}-\boldsymbol{q}|\right),
$$

where $K_{0}($.$) is the zeroth-order modified Bessel function. Since the gradient of b$ lies in the $\boldsymbol{e}_{x}$-direction we may identify the local coordinates as follows:

$$
(\xi, \eta)=\left(x-q_{1}, y-q_{2}\right)=(\rho \cos \theta, \rho \sin \theta), \quad \rho=\left(\xi^{2}+\eta^{2}\right)^{1 / 2} .
$$

Substituting for $x$ and $y$ in (3.3), then calculating the stream function using (3.2), and finally the flow around the vortex using (2.4) we find

$$
\boldsymbol{u}_{o}^{(0)}=\frac{\Gamma \alpha}{2} \exp \left(\frac{\alpha \xi}{2}\right)\left(K_{0}\left(\frac{\alpha}{2} \rho\right) \boldsymbol{e}_{2}+K_{1}\left(\frac{\alpha}{2} \rho\right) \boldsymbol{e}_{\theta}\right),
$$

where $\boldsymbol{e}_{\theta}$ is the unit vector in the azimuthal direction about the vortex. This has the following asymptotic behaviour as the vortex is approached:

$$
\boldsymbol{u}_{o}^{(0)} \sim \frac{\Gamma \boldsymbol{e}_{\theta}}{\rho}-\frac{\Gamma \alpha}{2} \log \rho \boldsymbol{e}_{2}+\frac{\Gamma \alpha}{2}\left(\left(\log \left(\frac{4}{\alpha}\right)-\gamma\right) \boldsymbol{e}_{2}+\cos \theta \boldsymbol{e}_{\theta}\right)+\cdots \quad \text { as } \quad \rho \rightarrow 0,
$$

where here $\gamma$ is Euler's constant. Comparing this with the expansion of $\boldsymbol{u}_{o}^{(0)}$ found in equation (2.28) we see that

$$
\boldsymbol{u}_{o, r e g}^{(0)}=\frac{\Gamma \alpha}{2}\left(\log \left(\frac{4}{\alpha}\right)-\gamma+1\right) \boldsymbol{e}_{y} .
$$

We can then substitute the above into equation (2.90) to obtain the velocity of a single vortex:

$$
\dot{\boldsymbol{q}}(t)=\frac{\Gamma \alpha}{2}\left(\log \left(\frac{4}{\epsilon \alpha}\left(\frac{b(\boldsymbol{q}(t))}{b(\boldsymbol{q}(0))}\right)^{1 / 2}\right)+\frac{\tilde{\mathscr{P}}}{\Gamma^{2}}-\gamma-\frac{1}{2}\right) \boldsymbol{e}_{y}+O(\epsilon \log \epsilon),
$$

where $\tilde{P}$ is defined in (2.87) and (2.86).

Where there are $n$ vortices present at positions $\boldsymbol{x}=\boldsymbol{q}^{j}(t)(j=1, \ldots, n)$ and with circulations $\Gamma_{j}$ we must solve equation (2.92) for the stream function. The solution to this equation is just the linear superposition of the $n$ single-vortex solutions for

$\dagger$ I am grateful to an anonymous referee for pointing out the existence of this exact solution to the flow problem. 
vortices at positions $\boldsymbol{x}=\boldsymbol{q}^{j}(j=1, \ldots, n)$ and with circulations $\Gamma_{j}$, that is

$$
\psi^{(0)}=H \exp \left(-\frac{\alpha x}{2}\right) \sum_{j=1}^{n} \Gamma_{j} \exp \left(-\frac{\alpha q_{1}^{j}}{2}\right) K_{0}\left(\frac{\alpha}{2}\left|\boldsymbol{x}-\boldsymbol{q}^{j}\right|\right) .
$$

We then use equation (2.91) to find the corresponding fluid velocity

$$
\begin{aligned}
\boldsymbol{u}_{o}^{(0)}=\frac{\alpha}{2} \sum_{j=1}^{n} \Gamma_{j} \exp \left(\frac{\alpha}{2}\left(x-q_{1}^{j}\right)\right)\left[K_{0}\left(\frac{\alpha}{2}\left|\boldsymbol{x}-\boldsymbol{q}^{j}\right|\right) \boldsymbol{e}_{y}\right. \\
\left.+K_{1}\left(\frac{\alpha}{2}\left|\boldsymbol{x}-\boldsymbol{q}^{j}\right|\right)\left(\frac{\left(x-q_{1}^{j}\right) \boldsymbol{e}_{y}-\left(y-q_{2}^{j}\right) \boldsymbol{e}_{x}}{\left|\boldsymbol{x}-\boldsymbol{q}^{j}\right|}\right)\right],
\end{aligned}
$$

and carry out the same procedure as we did for the single vortex; that is we expand $\boldsymbol{u}_{o}^{(0)}$ about the $k$ th vortex say, compare this with expansion (2.94) to calculate $\left(\boldsymbol{u}_{o, \text { reg }}^{(0)}\right)_{k}$ and then substitute into (2.95) to obtain the velocity of the $k$ th vortex,

$$
\begin{aligned}
\dot{\boldsymbol{q}}^{k}(t)= & \frac{\Gamma_{k} \alpha}{2}\left(\log \left(\frac{4}{\epsilon \alpha}\left(\frac{b\left(\boldsymbol{q}^{k}(t)\right)}{b\left(\boldsymbol{q}^{k}(0)\right)}\right)^{1 / 2}\right)+\frac{\tilde{P}_{k}}{\Gamma_{k}^{2}}-\gamma-\frac{1}{2}\right) \boldsymbol{e}_{y} \\
& +\frac{\alpha}{2} \sum_{j \neq k}^{n} \Gamma_{j} \exp \left(\frac{\alpha}{2}\left(q_{1}^{k}-q_{1}^{j}\right)\right)\left[K_{0}\left(\frac{\alpha}{2}\left|\boldsymbol{q}^{k}-\boldsymbol{q}^{j}\right|\right) \boldsymbol{e}_{y}\right. \\
& \left.+K_{1}\left(\frac{\alpha}{2}\left|\boldsymbol{q}^{k}-\boldsymbol{q}^{j}\right|\right)\left(\frac{\left(q_{1}^{k}-q_{1}^{j}\right) \boldsymbol{e}_{y}-\left(q_{2}^{k}-q_{2}^{j}\right) \boldsymbol{e}_{x}}{\left|\boldsymbol{q}^{k}-\boldsymbol{q}^{j}\right|}\right)\right]+O(\epsilon \log \epsilon) .
\end{aligned}
$$

An example of the motion of two vortices, of equal strength but opposite circulations $\Gamma=1$ and $\Gamma=-1$, over the exponential depth profile $b=\exp (-x)$ is given in figure 2. Here the vortices move from initially shallow water into deeper water, coming very close together, and accelerating, as they do so.

\section{Numerical solution of the model}

In this section we shall illustrate the law of motion derived in $\S 2$ with two examples of vortex motion. For simplicity we take the depth to be a function of $x$ only, $b=b(x)$. We can thus identify $x-q_{1}(t)$ with $\xi$ and $y-q_{2}(t)$ with $\eta$ throughout the motion.

A single vortex. We begin by solving for the leading-order outer flow around a single vortex at position $\boldsymbol{x}=\left(q_{1}(t), q_{2}(t)\right)$. In order to do this we use the stream function formulation given by equations (2.4) and (2.30). It proves convenient to split the stream function into two parts:

$$
\psi^{(0)}=-\frac{\Gamma}{2} \log \left(|\boldsymbol{x}-\boldsymbol{q}(t)|^{2}\right)\left(b_{0}+\frac{B_{1}\left(x-q_{1}(t)\right)}{2}\right)+A(x, y) .
$$

Here the first part of the stream function leads to the unbounded terms in $\boldsymbol{u}_{o}^{(0)}$ on the vortex, and $b_{0}$ and $B_{1}$ are (as before) the coefficients of the first two terms in the expansion of the depth $b$ about the vortex,

$$
b \sim b_{0}+B_{1}\left(x-q_{1}(t)\right)+\cdots \quad \text { as } \quad|\boldsymbol{x}-\boldsymbol{q}(t)| \rightarrow 0 .
$$




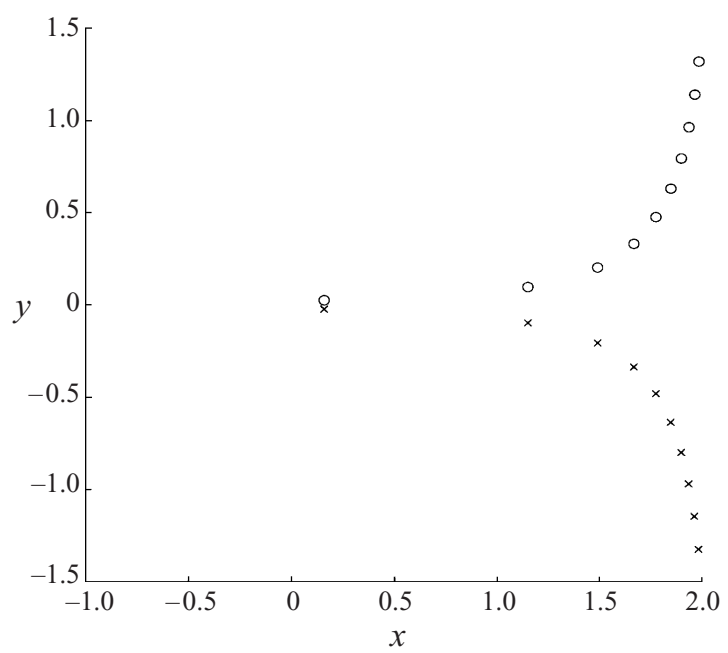

FIGURE 2. The motion of a pair of vortices of equal strength but with circulations of opposite signs over the profile $b=\exp (-x)$ : the positions of the vortices (of strengths $\Gamma=1(\times)$ and $\Gamma=-1(O))$ are given at evenly spaced times 0.1 units apart, starting from $(x, y)=(2.0,-1.5)$ and $(x, y)=(2.0,1.5)$. The vortices are assumed initially to have uniform vorticity (strength $\left.\omega_{i}=1 / \epsilon^{2}\right)$ contained within a circle radius $\epsilon$ (where $\epsilon=0.1$ ). This assumption leads to a value of the vortex structure constant $\tilde{P}=5 \Gamma^{2} / 4$. The flow around the vortices is calculated analytically in $\S 3$ and is used to determine the vortex velocities.

Substituting in (2.30) we find the following equation for $A$ :

$$
\begin{aligned}
\nabla \cdot\left(\frac{1}{b} \nabla A\right)=-\frac{\Gamma}{4 b^{2}}[ & B_{1} b_{x} \log \left(|\boldsymbol{x}-\boldsymbol{q}(t)|^{2}\right) \\
& \left.+\frac{2 b_{x} B_{1}\left(x-q_{1}(t)\right)^{2}+4\left(b_{0} b_{x}-b B_{1}\right)\left(x-q_{1}(t)\right)}{|\boldsymbol{x}-\boldsymbol{q}(t)|^{2}}\right],
\end{aligned}
$$

which, given appropriate boundary conditions, we can solve numerically. Note that the second term on the right-hand side of this equation, when expanded about the point $(x, y)=\left(q_{1}, q_{2}\right)$ (where we use the local coordinates defined in (2.11)) has the asymptotic behaviour

$$
\frac{2 b_{x} B_{1}\left(x-q_{1}(t)\right)^{2}+4\left(b_{0} b_{x}-b B_{1}\right)\left(x-q_{1}(t)\right)}{|\boldsymbol{x}-\boldsymbol{q}(t)|^{2}} \sim \text { const. } \cos ^{2} \theta .
$$

The leading term in the expansion of the right-hand side of equation (4.3) is thus the first term, which has a $\log \rho$ singularity. If we introduce a cutoff around this point of a size comparable to the size of the grid used to solve this equation $h$, then the error induced will be of $O\left(h^{2} \log h\right)$.

We now need to calculate $\boldsymbol{u}_{o, r e g}^{(0)}$ (the regular part of the fluid velocity at the vortex) from the solution of (4.3). Using (4.1) and (2.4) we derive an expression for $b \boldsymbol{u}_{o}^{(0)}$ about the vortex, at $\boldsymbol{x}=\boldsymbol{q}(t)$, in terms of the local coordinates defined in $\S 2$ :

$$
b \boldsymbol{u}_{o}^{(0)} \sim \Gamma\left(\frac{b_{0} \boldsymbol{e}_{\theta}}{\rho}+\frac{B_{1}}{2}\left(\log \rho \boldsymbol{e}_{2}+\cos \theta \boldsymbol{e}_{\theta}\right)\right)+\left.\left(\frac{\partial A}{\partial \eta} \boldsymbol{e}_{1}-\frac{\partial A}{\partial \xi} \boldsymbol{e}_{2}\right)\right|_{x=q(t)}+O(\rho \log \rho) .
$$


Multiplying the expansion for $\boldsymbol{u}_{o}^{(0)}$, found in (2.28), by the corresponding expansion of $b\left(b=b_{0}+B_{1} \rho \cos \theta+\cdots\right)$ and comparing the result with (4.5) gives rise to the following expression for the regular part of the fluid velocity at the vortex:

$$
\boldsymbol{u}_{o, r e g}^{(0)}=\left.\frac{1}{b_{0}}\left(\frac{\partial A}{\partial y} \boldsymbol{e}_{x}-\frac{\partial A}{\partial x} \boldsymbol{e}_{y}\right)\right|_{x=q(t)}-\frac{\Gamma B_{1}}{2 b_{0}} \boldsymbol{e}_{y} .
$$

Using equation (2.90) we can now write down the velocity of a single vortex; it is

$$
\begin{aligned}
\dot{\boldsymbol{q}}=-\frac{B_{1} \Gamma}{2 b_{0}}\left(\log \left(\frac{1}{\epsilon}\left(\frac{b(\boldsymbol{q}(T))}{b(\boldsymbol{q}(0))}\right)^{1 / 2}\right)+\frac{\tilde{P}}{\Gamma^{2}}-\frac{1}{2}\right) \boldsymbol{e}_{y} & \\
& +\left.\frac{1}{b_{0}}\left(\frac{\partial A}{\partial y} \boldsymbol{e}_{x}-\frac{\partial A}{\partial x} \boldsymbol{e}_{y}\right)\right|_{x=q(t)}+O(\epsilon \log \epsilon) .
\end{aligned}
$$

The symmetry of the problem forces $\left.A_{y}\right|_{x=q(t)}=0$. It follows that an isolated vortex will move along a contour of the bottom topography, the direction of motion being determined by the sign of its circulation.

Many vortices. Where there are $n$ vortices with circulations $\Gamma_{j}$ at positions $\boldsymbol{x}=\boldsymbol{q}^{j}(t)$ $(j=1, \ldots, n)$ the stream function of the flow $\psi^{(0)}$ satisfies equation (2.31). This stream function is simply the linear superposition of the $n$ stream functions $\psi_{j}^{(0)}$ resulting from placing a solitary vortex of circulation $\Gamma_{j}$ at each of the positions $\boldsymbol{x}=\boldsymbol{q}^{j}$; in other words

$$
\psi^{(0)}(\boldsymbol{x}, t)=\sum_{j=1}^{n} \psi_{j}^{(0)}(\boldsymbol{x}, t), \quad \text { where } \quad \nabla \cdot\left(\frac{1}{b} \nabla \psi_{j}^{(0)}\right)=-2 \pi \Gamma_{j} \delta\left(\boldsymbol{x}-\boldsymbol{q}^{j}(t)\right) .
$$

We can solve $(4.8 b)$ for each $j$ as in the case of the single vortex (this scenario is discussed at the beginning of this section). Then, in order to find the regular part of the fluid flow at $\boldsymbol{x}=\boldsymbol{q}^{k}(t)$ (the position of the $k$ th vortex), we need to calculate

$$
\begin{gathered}
A^{k}(\boldsymbol{x})=a^{k}(\boldsymbol{x}, t)+\sum_{j \neq k}^{n} \psi_{j}^{(0)}(\boldsymbol{x}, t), \\
\nabla \cdot\left(\frac{1}{b} \nabla a^{k}\right)=-\frac{\Gamma_{k}}{4 b^{2}}\left[B_{1} b_{x} \log \left(\left|\boldsymbol{x}-\boldsymbol{q}^{k}(t)\right|^{2}\right)\right. \\
\left.+\frac{2 b_{x} B_{1}\left(x-q_{1}^{k}(t)\right)^{2}+4\left(b_{0} b_{x}-b B_{1}\right)\left(x-q_{1}^{k}(t)\right)}{\left|\boldsymbol{x}-\boldsymbol{q}^{k}(t)\right|^{2}}\right] .
\end{gathered}
$$

The velocity of the $k$ th vortex is then given by equation (4.7), where we replace $A$ by $A^{k}$.

\subsection{Examples of the motion of two interacting vortices}

In the following we examine the interaction of two vortices in the presence of a onedimensional topography. For definiteness we take the initial distribution of vorticity to be uniform within a distance $\epsilon$ of their centres, and zero outside. With this assumption we calculate $\tilde{P}=5 \Gamma^{2} / 4$. We shall also take $\epsilon=0.1$. 

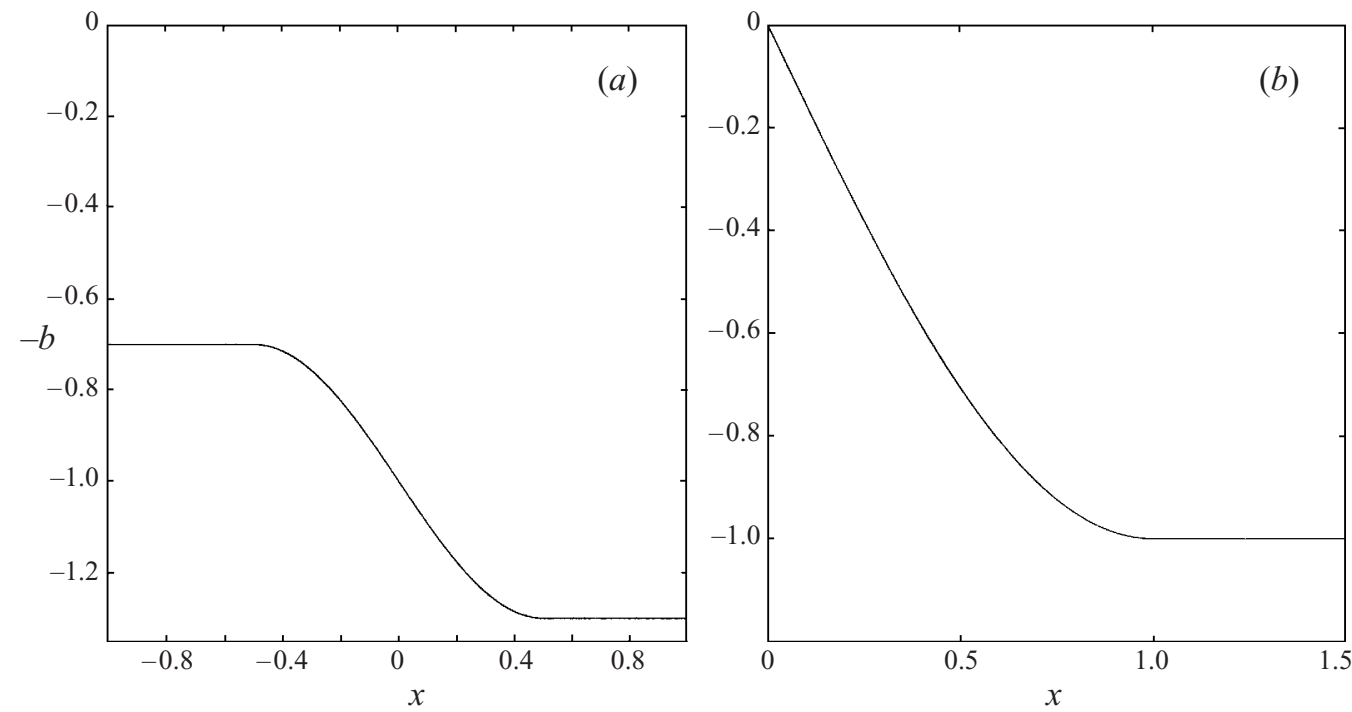

FIgURE 3. Profiles of the depth of the step (a) (see figure 5) and of the shore (b) (see figure 6) are plotted.

The smooth step. We start by considering the depth profile plotted in figure $3(a)$. Before attempting a solution of (4.9) we need to decide upon sensible boundary conditions for $\psi^{(0)}$ (and hence $A$ ). Providing we solve in a sufficiently large domain we can impose far-field conditions which are asymptotically correct (in inverse powers of the size of the domain) when viewed from a sufficient distance. In this particular case the far-field problem is a sharp step with jump along the $y$-axis. In other words, we write

$$
x=\lambda X, \quad y=\lambda Y, \quad \boldsymbol{q}=\lambda \boldsymbol{Q},
$$

where $\lambda \gg 1$ is the size of our numerical domain, and look for a solution to the single-vortex problem (2.30), i.e.

$$
\frac{\partial}{\partial X}\left(\frac{1}{b(X / \lambda)} \frac{\partial \psi^{(0)}}{\partial X}\right)+\frac{1}{b(X / \lambda)} \frac{\partial^{2} \psi^{(0)}}{\partial Y^{2}}=-2 \pi \Gamma \lambda^{2} \delta\left(\lambda\left(X-Q_{1}\right)\right) \delta\left(\lambda\left(Y-Q_{2}\right)\right) .
$$

Solving this at leading order and transferring back to the unstretched variables $(x, y)$ we see that, where $q_{1}>0$, the solution $\psi^{(0)}$ to $(2.30)$ has the following asymptotic behaviour for large $|\boldsymbol{x}|$ :

$$
\begin{gathered}
\psi^{(0)} \sim-\frac{\Gamma \mathscr{B}_{+} \mathscr{B}_{-}}{\left(\mathscr{B}_{+}+\mathscr{B}_{-}\right)} \log \left(|\boldsymbol{x}-\boldsymbol{q}|^{2}\right), \quad x<0, \\
\psi^{(0)} \sim-\frac{\Gamma \mathscr{B}_{+}}{2}\left(\log \left(|\boldsymbol{x}-\boldsymbol{q}|^{2}\right)-\left(\frac{\mathscr{B}_{+}-\mathscr{B}_{-}}{\mathscr{B}_{+}+\mathscr{B}_{-}}\right) \log \left(\left(\left(x+q_{1}\right)^{2}+\left(y-q_{2}\right)^{2}\right)\right)\right), \quad x>0,
\end{gathered}
$$

where $\mathscr{B}_{+}=\lim _{x \rightarrow \infty} b(x)$ and $\mathscr{B}_{-}=\lim _{x \rightarrow-\infty} b(x)$. Similarly one can calculate the 

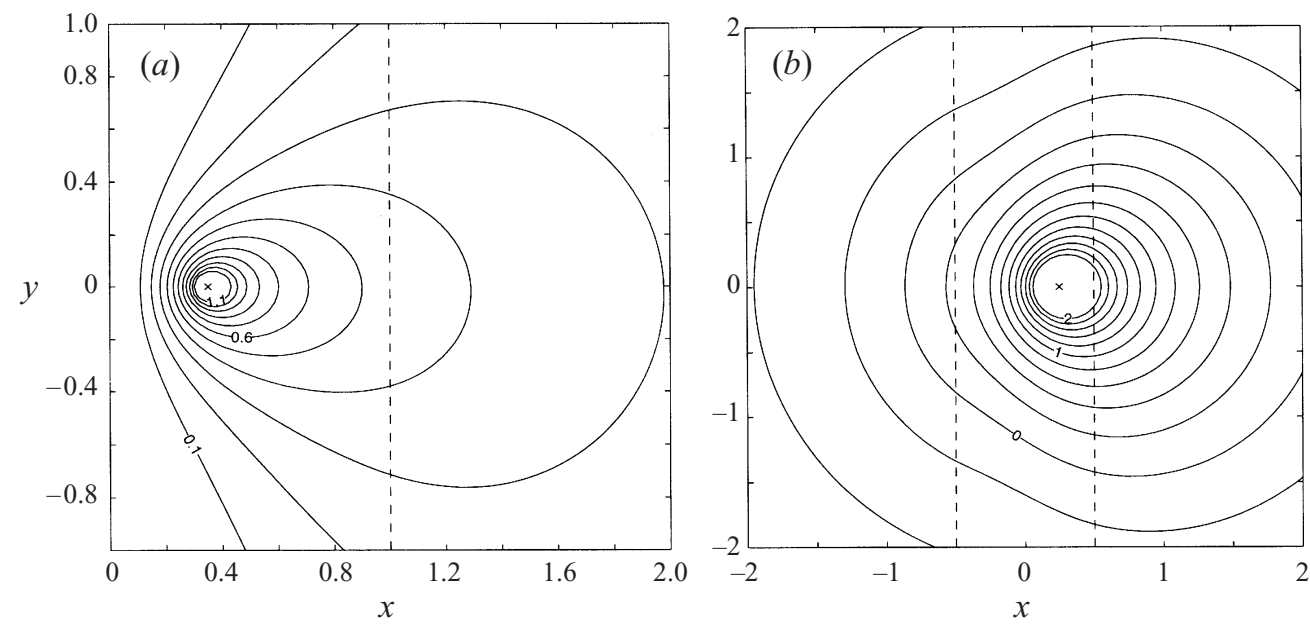

FiguRE 4. Examples of the stream functions calculated for a single vortex of circulation $\Gamma=1$. In (a) the step profile (see figure $3 a)$ is used and the vortex is placed at $(x, y)=(0.25,0)$ while in $(b)$ the beach profile (see figure $3 b$ ) is used for the depth (see figure $3 b$ ) and the vortex is placed at $(x, y)=(0.35,0)$. In both pictures the position of the vortex is given by a ' $x$ ' and stream lines close to the vortex have been ommitted for clarity.

far-field behaviour for $q_{1}<0$ which is as follows:

$$
\begin{aligned}
\psi^{(0)} \sim- & \frac{\Gamma \mathscr{B}_{-}}{2}\left(\log \left(|\boldsymbol{x}-\boldsymbol{q}|^{2}\right)\right. \\
+ & \left.\left(\frac{\mathscr{B}_{+}-\mathscr{B}_{-}}{\mathscr{B}_{+}+\mathscr{B}_{-}}\right) \log \left(\left(\left(x+q_{1}\right)^{2}+\left(y-q_{2}\right)^{2}\right)\right)\right), \quad x>0, \\
& \psi^{(0)} \sim-\frac{\Gamma \mathscr{B}_{+} \mathscr{B}_{-}}{\left(\mathscr{B}_{+}+\mathscr{B}_{-}\right)} \log \left(|\boldsymbol{x}-\boldsymbol{q}|^{2}\right), \quad x<0 .
\end{aligned}
$$

These results are very similar to some derived by Bidlot $\&$ Stern (1994) for the stream function over a step. However they use a constant-depth stream function and match this over the step by continuity arguments.

We can use this far-field behaviour as a boundary condition on equation (2.30) for the single vortex. In order to solve this equation it is convenient to split $\psi^{(0)}$ up into a singular part and a (more or less) regular part $A(x, y) . \dagger$ We can solve for $A(x, y)$ from equation (4.3) by applying a standard numerical technique for the solution of elliptic PDEs and truncating the singular terms on the right-hand side in a small region surrounding the vortex (it can be shown that this only introduces a small error). The boundary conditions imposed on $A(x, y)$ on the edge of the numerical domain must be consistent with (4.13)-(4.16). An example of the stream function $\psi^{(0)}$ calculated using this method for the step geometry is shown in figure $4(a)$.

For the two-vortex problem we can calculate $A^{1}$ and $A^{2}$ by linearly superimposing solutions of two single-vortex problems as discussed for equations (4.9) and (4.10). We can then calculate the velocities of vortex 1 and vortex 2 by subsituting $A^{1}(x, y)$ and $A^{2}(x, y)$ respectively into equation (4.7) in place of $A$. A simple time-stepping procedure (forward Euler) can be used to calculate the positions of the vortices at the next time step. The results of calculations using this method, which show the motion 

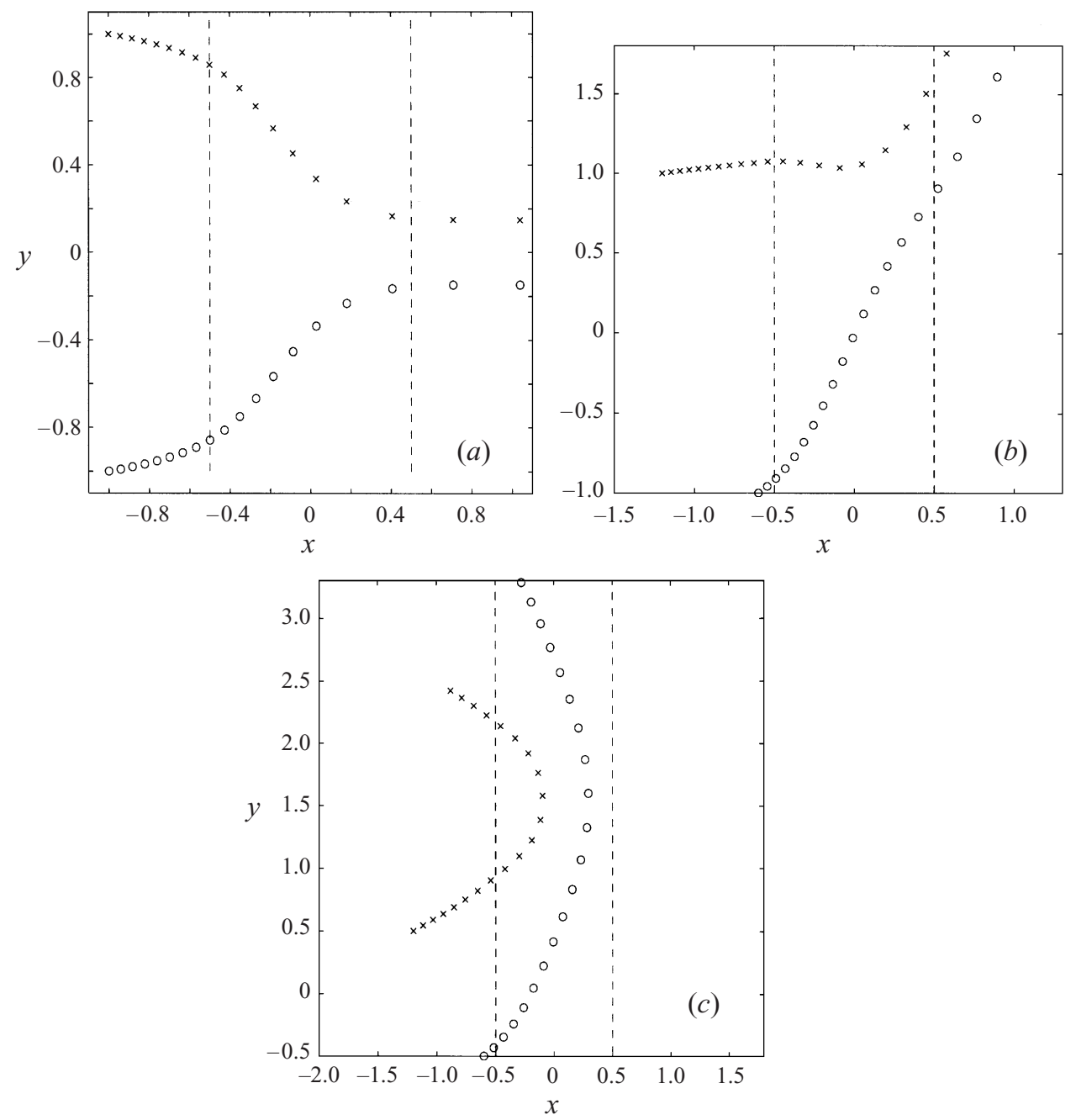

FIGURE 5. The motion of a pair of vortices of equal strength but with circulations of opposite signs over a step for three different initial conditions. The profile of this step is plotted in figure $3(a)$. The positions of the vortices (of strengths $\Gamma=1(\times)$ and $\Gamma=-1(O)$ ) are given at evenly spaced times 0.1 units apart are shown. In these pictures the dashed lines mark the extent of the step. The vortices are assumed initially to have uniform vorticity (strength $\omega_{i}=1 / \epsilon^{2}$ ) contained within a circle radius $\epsilon$ (where $\epsilon=0.1$ ). This assumption leads to a value of the vortex structure constant $\tilde{\mathscr{P}}=5 \Gamma^{2} / 4$.

of vortex pairs of equal strength but with circulations of opposite sign, are shown in figure 5.

The one-dimensional shoreline. We can use the procedure outlined above to calculate the motion of vortices along the one-dimensional shoreline given in figure $3(b)$. There are however some differences; one of these is the boundary condition which should be applied to (4.9). For the single-vortex problem (2.30) an appropriate boundary condition on the edge of the computational domain $\left(x^{2}+y^{2} \gg 1, x>0\right)$ is

$$
\psi^{(0)} \sim-\frac{\Gamma \mathscr{B}_{+}}{2}\left(\log \left(\left(x-q_{1}\right)^{2}+\left(y-q_{2}\right)^{2}\right)-\log \left(\left(x+q_{1}\right)^{2}+\left(y-q_{2}\right)^{2}\right)\right),
$$



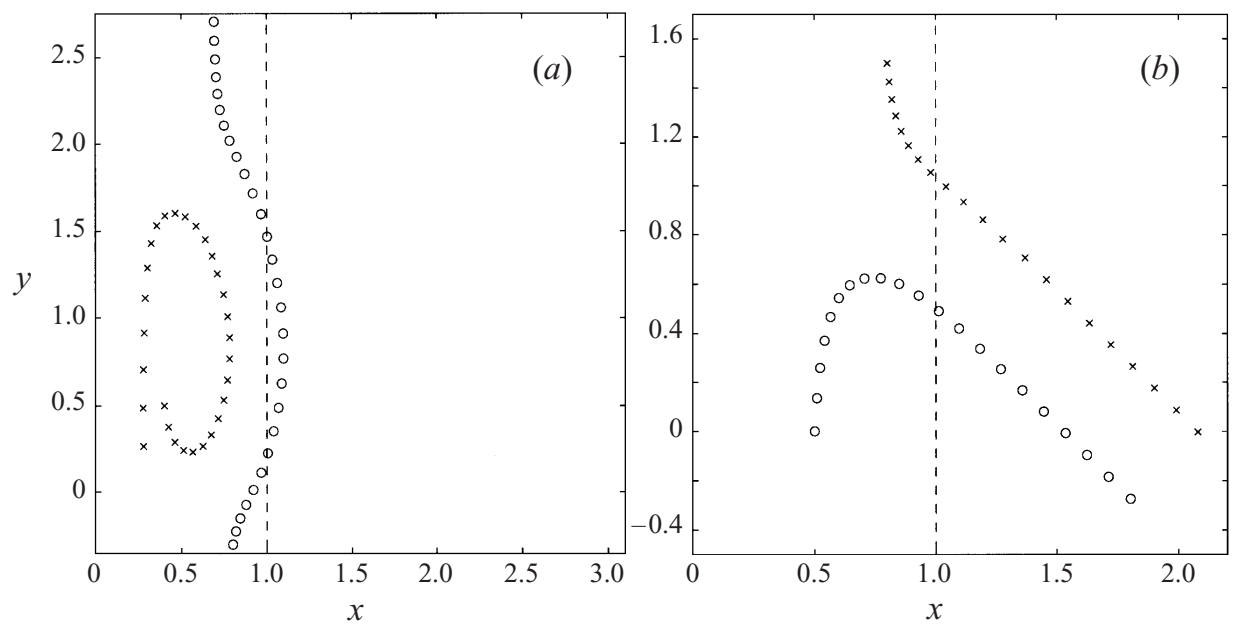

FIGURE 6. The motion of an equal and opposite vortex pair along a shoreline. The profile of this shoreline is plotted in figure $3(b)$. The positions of the vortices (of strengths $\Gamma=1(\times)$ and $\Gamma=-1$ $(O))$ are given at evenly spaced times 0.05 units apart are shown. In $(a)$ the positive vortex $(\times)$ starts from position $(0.4,0.5)$ and the negative one at the bottom of the picture. The same assumption about the initial distribution of vorticity within the core is used as used in figure 5 and it follows that here $\epsilon=0.1$ and $\tilde{\mathscr{P}}=5 \Gamma^{2} / 4$.

where $\mathscr{B}_{+}=\lim _{x \rightarrow \infty} b(x)$. There is also the question of how to deal with the singularity in $1 / b$ as $x \rightarrow 0$. An asymptotic analysis in the vicinity of this line reveals that

$$
\psi^{(0)} \sim \text { const. }+O\left(x^{2}\right) \quad \text { as } x \rightarrow 0 .
$$

This suggests that we should apply the boundary condition $\psi^{(0)}=$ const. on $x=\delta$. Here $\delta \ll 1$ and the constant is chosen to ensure continuity with the far-field boundary condition. An example of the stream function $\psi^{(0)}$ calculated for the shoreline geometry is shown in figure $4(b)$.

As before, once we have found single-vortex solutions, each centred at one of the vortices, we can construct solutions to (4.9) and hence calculate the vortex velocities. Figure 6 shows the results of calculations for vortex pairs moving along the onedimensional shoreline of figure $3(b)$.

\subsection{Discussion of the motion of vortex pairs over topography pictured in figures 4-6}

Figure 5 shows the motion of two vortices, each with uniform vorticity contained in a circle radius of $\epsilon=0.1$, over the smooth step whose profile is plotted in figure $3(a)$. The extent of the step in figure 5 is shown by the dashed lines with shallow water of depth $b=0.7$ to the left of these and deeper water of depth $b=1.3$ to the right. In figure 5(a) the vortices initially start two units, or 20 vortex radii, apart with their line of centres parallel and to the left of the step. Initially they both move to the right, under the action of the other vortex, being advected in each other's flow. There is also a slight drift together which can be explained by the interaction of each vortex with the global topography. In some sense the step, which is still some distance from the vortex, gives rise to an image vortex, with the same sign circulation as the real vortex but of weaker strength. This causes the motion in the $y$-direction. To check this statement it is helpful to look at equations (4.15) and (4.16) which are an exact solution to the sharp step problem with a single vortex situated to the right of the step lying along the line $x=0$. As the vortices approach the step their drift together 
accelerates under the increasingly strong action of their images. When they hit the step the $O(\log (1 / \epsilon))$ velocity term, resulting from the local gradient in the depth $\nabla b$ at the vortex, appears. This acts to drive the vortices together in the $y$-direction but as it decreases the distance between the vortices so the strength of the vortex pair interaction increases and causes an acceleration in the $x$-direction. After clearing the step the pair continues its motion in the $x$-direction but with a smaller distance between them, and hence a greater velocity than before the interaction with the step.

Figures $5(b)$ and $5(c)$ show similar evolutions over the same step profile. The difference between the subsequent motions is explained by the different initial conditions. In figure 4(b) there is a slight angle between the initial line of centres and the step. This causes the vortex pair to be deflected upwards as it crosses the step and occurs because the lower vortex comes into contact with the step before the upper one. In figure $5(c)$ the initial angle of the line of centres of the vortices to the step is greater than in figure $5(b)$ and the subsequent deflection of the pair is large enough to cause it to alter its course (which initially seemed to be taking them it into deep water) back into the shallow water in the left of the picture.

In figure 6 we show the evolution of vortex pairs (again with a uniform vorticity profile contained within a radius of 0.1 of their centres) over a beach whose profile is plotted in figure $3(b)$. To the right of the dashed line $x=1.0$ (in figure 6) the profile flattens off to a uniform depth $b=1.0$, while along the line $x=0$ the depth $b=0$. Figure $6(a)$ shows a negative vortex, initially at the bottom of the picture and moving in the positive $y$-direction (up the beach), interacting with a positive vortex which starts at the point $(0.4,0.5)$ and is initially moving down the beach. The vortices move together under the action of the gradient in the depth until the interaction between the two vortices (advection in each other's flow fields) becomes comparable with, and even dominates, the effect of the sloping beach. The vortices then move up the beach as a pair (in the positive $y$-direction) until the negative vortex overtakes the positive one. After which point they separate with the positive vortex returning down the beach, under the action of the slope, while the negative one continues up it. In $(b)$ a different starting configuration ensures that the two vortices, which come together under the action of $\nabla b$, move out into deep water under their mutual interaction as a vortex pair.

Needless to say these examples of vortex pair interaction over one-dimensional topographies give a very limited idea of the possible vortex interactions with topography and other vortices which could be described by the model derived in this paper. They are presented here in order to illustrate the model and not in the spirit of a detailed investigation of possible vortex motions over topography.

\section{Conclusion}

We have derived a law of motion for well-separated vortices in shallow inviscid fluid of varying depth in the distinguished limit as the Froude number $\mathscr{F}$, flow aspect ratio $v$ and the core radius $\epsilon$ tend to zero with $\epsilon \gg \mathscr{F}$ and $\epsilon \gg v$. At leading order we found that a vortex of circulation $\Gamma$ is driven by the local gradient in the logarithm of the depth $b$ along a contour of the bottom with velocity

$$
\dot{\boldsymbol{q}}=\left.\frac{\Gamma}{2} \log \left(\frac{1}{\epsilon}\right) \nabla \log b\right|_{x=q(t)} \wedge \boldsymbol{e}_{3}+O(1) .
$$

It was then shown that the order-one correction to this law depends upon the global bottom topography, the position of other vortices and the vortex core structure. 
The method for calculating the vortex velocity correct to the two leading orders is summarized in $\S 2.3$ in equations (2.91)-(2.98).

Although, in our derivation, we have assumed that vortices are separated by $O(1)$ distances the asymptotics are robust enough to cover cases in which the typical vortex separation is of $O(\delta)$ where $\epsilon \ll \delta \ll 1$. In such circumstances the term in the vortex velocity arising from vortex interaction is of $O(1 / \delta)$ and thus may be comparable to, or bigger than, that arising from gradients in the depth.

We then proposed a numerical method, which takes account of the two leading terms in the law of motion, for solving vortex interaction problems in the presence of variations in the bottom topography. This method was used to investigate the motion of a vortex pair. Our simulations show that, while motion resulting from gradients in the depth dominates for sufficiently large distance between vortices, interesting effects occur at shorter distances; these result from the competition between topographydriven motion and motion arising from vortex interactions. In this context we note that a sloping bottom can have the effect of bringing vortices of an opposite sign closer together and induce them to move out to deeper water. While the distance between these remains a large number of vortex diameters they remain as separate coherent structures. However if the action of the slope brings them into sufficiently close proximity we conjecture that they may form a nonlinear vortex couple with core structure described in Batchelor $(1967, \S 7.3)$. From a practical point of view this may be of some importance because it is thought that vortex couples play an important part in the transport of sediments, pollutants, etc. offshore (Peregrine 1995). Indeed they may be the major factor in the removal of sand from a beach in a storm.

I am grateful to D. D. Holm for bringing this problem to my attention and for some useful comments and would like to thank J. Rubinstein and L. J. Cummings for their comments on the substance and presentation of this work. I would also like to thank anonymous referees for helpful suggestions on the content of the paper. The research was funded in part by the Koret Research foundation and in part by the E.U.

\section{REFERENCES}

Allen, J. S., Newberger, P. A. \& Holman, R. A. 1996 Nonlinear shear instabilities of alongshore currents on plane beaches. J. Fluid Mech. 310, 181-213.

Batchelor, G. K. 1967 An Introduction to Fluid Dynamics. Cambridge University Press.

Bidlot, J. R. \& Stern, M. E. 1994 Maintenance of continental boundary-layer shear flow through counter-gradient vorticity flux in a barotropic model. J. Fluid Mech. 271, 55-85.

Callegari, A. J. \& Ting, L. 1978 Motion of a curved vortex filament with decaying vortical core and axial velocity. SIAM J. Appl. Maths 35, 148-175.

Cammasa, R., Holm, D. D. \& Levermore, C. D. 1996 Long-time effects of bottom topography in shallow water. Physica D 98, 258-286.

Chapman, S. J. \& Richardson, G. 1997 Vortex pinning by inhomogeneities in type-II superconductors. Physica D 108, 397-407.

Couder, Y. \& Basdenvant, C. 1986 Experimental and numerical study of vortex couples in two dimensional flows. J. Fluid Mech. 173, 225-251.

Grimshaw, R. \& YI, Z. X. 1991 Evolution of a potential vorticity front over a topographic slope. J. Phys. Oceanogr. 21, 1240-1255.

Hamm, L., Masden, P. A. \& Peregrine, D. H. 1993 Wave transformations in the nearshore zone: a review. Coastal Engng 21, 5-39.

Mariotti, A., Legras, B. \& Dritschel, D. G. 1994 Vortex stripping and the erosion of coherent vortex structures in two-dimensional flows. Phys. Fluids 6, 3954-3962.

Moore, D. W. \& Saffman, P. G. 1972 The motion of a vortex filament with axial flow. Phil. Trans. R. Soc. Lond. A 272, 403-429. 
Oltman-Shay, J., Howd, P. A. \& Birkemeier, W. A. 1989 Shear instabilities of the mean longshore current 2. field observations. J. Geophys. Res. 94, C12, 18031-18042.

Peregrine, D. H. 1995 Vorticity and eddies in the surf zone. Proc. Coastal Dynamics, Gyndia.

ReZniK, G. M. \& Dewar, W. K. 1994 An analytic theory of distributed axisymmetric barotropic vortices on the $\beta$-plane. J. Fluid Mech. 269, 301-321.

Slinn, D. N., Allen, J. S., Newberger, P. A. \& Holman, R. A. 1998 Nonlinear shear band instabilities of alongshore currents over barred beaches. J. Geophys. Res. 103, C9, 1835718379.

Signell, R. P. \& Geyer, W. R. 1991 Transient eddy formation around headlands. J. Geophys. Res. 96, C2, 2561-2575.

Sutyrin, G. G. \& Fleirl, G. R. 1994 Intense vortex motion on the $\beta$ plane: development of $\beta$-gyres. J. Atmos. Sci. 51, 773-790.

Van Dyke, M. 1975 Perturbation Methods in Fluid Dynamics, §5. Parabolic Press.

Van Dyke, M. 1982 An Album of Fluid Motion. Parabolic Press. 